\title{
Dynamics of Convectively Driven Banded Jets in the Laboratory
}

\section{Citation}

Read, Peter L., Yasuhiro H. Yamazaki, Stephen R. Lewis, Paul D. Williams, Robin Wordsworth, Kuniko Miki-Yamazaki, Joël Sommeria, and Henri Didelle. 2007. "Dynamics of Convectively Driven Banded Jets in the Laboratory." Journal of the Atmospheric Sciences 64 (11) (November): 4031-4052. doi:10.1175/2007jas2219.1. http://dx.doi.org/10.1175/2007JAS2219.1.

\section{Published Version}

doi:10.1175/2007JAS2219.1

\section{Permanent link}

http://nrs.harvard.edu/urn-3:HUL.InstRepos:27846859

\section{Terms of Use}

This article was downloaded from Harvard University's DASH repository, and is made available under the terms and conditions applicable to Other Posted Material, as set forth at http:// nrs.harvard.edu/urn-3:HUL.InstRepos:dash.current.terms-of-use\#LAA

\section{Share Your Story}

The Harvard community has made this article openly available.

Please share how this access benefits you. Submit a story.

\section{Accessibility}




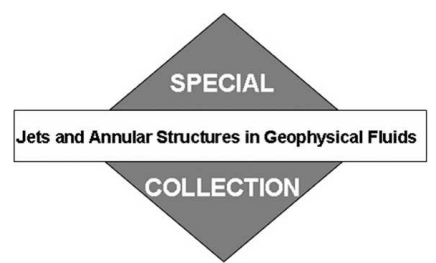

\title{
Dynamics of Convectively Driven Banded Jets in the Laboratory
}

\author{
Peter L. Read, Yasuhiro H. Yamazaki, Stephen R. Lewis,* Paul D. Williams, ${ }^{+}$ \\ Robin WordswOrTH, AND KUNIKO MIKI-YAMAZAKI \\ Clarendon Laboratory, Department of Physics, University of Oxford, Oxford, United Kingdom \\ Jö̈l SOMMERIA AND HENRI DiDELle \\ LEGI Coriolis, Grenoble, France
}

(Manuscript received 16 June 2006, in final form 12 January 2007)

\begin{abstract}
The banded organization of clouds and zonal winds in the atmospheres of the outer planets has long fascinated observers. Several recent studies in the theory and idealized modeling of geostrophic turbulence have suggested possible explanations for the emergence of such organized patterns, typically involving highly anisotropic exchanges of kinetic energy and vorticity within the dissipationless inertial ranges of turbulent flows dominated (at least at large scales) by ensembles of propagating Rossby waves. The results from an attempt to reproduce such conditions in the laboratory are presented here. Achievement of a distinct inertial range turns out to require an experiment on the largest feasible scale. Deep, rotating convection on small horizontal scales was induced by gently and continuously spraying dense, salty water onto the free surface of the 13-m-diameter cylindrical tank on the Coriolis platform in Grenoble, France. A "planetary vorticity gradient" or " $\beta$ effect" was obtained by use of a conically sloping bottom and the whole tank rotated at angular speeds up to $0.15 \mathrm{rad} \mathrm{s}^{-1}$. Over a period of several hours, a highly barotropic, zonally banded large-scale flow pattern was seen to emerge with up to 5-6 narrow, alternating, zonally aligned jets across the tank, indicating the development of an anisotropic field of geostrophic turbulence. Using particle image velocimetry (PIV) techniques, zonal jets are shown to have arisen from nonlinear interactions between barotropic eddies on a scale comparable to either a Rhines or "frictional" wavelength, which scales roughly as $\left(\beta / U_{\text {rms }}\right)^{-1 / 2}$. This resulted in an anisotropic kinetic energy spectrum with a significantly steeper slope with wavenumber $k$ for the zonal flow than for the nonzonal eddies, which largely follows the classical Kolmogorov $k^{-5 / 3}$ inertial range. Potential vorticity fields show evidence of Rossby wave breaking and the presence of a "hyperstaircase" with radius, indicating instantaneous flows that are supercritical with respect to the Rayleigh-Kuo instability criterion and in a state of "barotropic adjustment." The implications of these results are discussed in light of zonal jets observed in planetary atmospheres and, most recently, in the terrestrial oceans.
\end{abstract}

\section{Introduction}

The banded organization of clouds and zonal winds in the atmospheres of the outer planets has long fascinated atmosphere and ocean dynamicists and plan-

\footnotetext{
* Current affiliation: Department of Physics and Astronomy, The Open University, Milton Keynes, United Kingdom.

+ Current affiliation: Department of Meteorology, University of Reading, Reading, United Kingdom.
}

Corresponding author address: Peter L. Read, Clarendon Laboratory, Department of Physics, University of Oxford, Parks Road, Oxford, OX1 3PU, United Kingdom.

E-mail: p.read1@physics.ox.ac.uk etologists, especially with regard to the stability and persistence of these patterns. This banded organization, mainly apparent in clouds thought to be of ammonia and $\mathrm{NH}_{4} \mathrm{SH}$ ice, is one of the most striking features of the atmosphere of Jupiter. The cloud bands are associated with multiple zonal jets of alternating sign with latitude (Limaye 1986; Simon 1999; García-Melendo and Sánchez-Lavega 2001; Porco et al. 2003), with zonal velocities in excess of $100 \mathrm{~m} \mathrm{~s}^{-1}$ and widths of order $10^{4}$ $\mathrm{km}$. Similar patterns of multiple zonal jets are also found in the atmospheres of Saturn (Sánchez-Lavega et al. 2000; Porco et al. 2005; Sánchez-Lavega et al. 2006) and the other gas giants, though on different absolute scales.

A similar pattern of zonation may also occur in the

DOI: $10.1175 / 2007 J A S 2219.1$ 
earth's oceans, though on a very different scale to those of the gas giant planets. Patterns of zonally banded flow are fairly ubiquitous in numerical model simulations of ocean circulation carried out with sufficient horizontal resolution. In practice this seems to require a resolution of at least $16^{\circ}$ in latitude and longitude, within which banded flows appear on a scale of $2^{\circ}-4^{\circ}$ (Galperin et al. 2004; Maximenko et al. 2005; Richards et al. 2006), which are coherent in the east-west directions for 1000 $\mathrm{km}$ or more. Such features often appear to be surfaceintensified, but extend coherently to great depths in an equivalent-barotropic form. The observational evidence for this phenomenon is still quite sketchy, however, though similar features are now becoming apparent in suitably filtered satellite altimeter data (Maximenko et al. 2005), at least in medium-long-term time averages.

The dynamical origin of these banded structures remains poorly understood, although the fact that the jets decay with height in the upper tropospheres and lower stratospheres of Jupiter and Saturn would suggest that they are driven by momentum sources in the weakly stratified region in the atmosphere below the visible clouds. The depth to which this pattern of winds penetrates below the clouds remains controversial, but most approaches toward understanding the processes organizing the flow into zonal bands have suggested that the pattern may originate from the anisotropy in a shallow turbulent layer of fluid due to the $\beta$ effect, that is, owing to the latitudinal variation of the effective planetary vorticity (see, e.g., Ingersoll et al. 2004 and Vasavada and Showman 2005).

Until recently, quantitative understanding of this process has been based principally on high-resolution numerical simulations of two-dimensional or geostrophic turbulence in stirred rotating fluids (Williams 1978, 1979; Vallis and Maltrud 1993; Chekhlov et al. 1996; Galperin et al. 2001, 2006; Huang et al. 2001; Sukoriansky et al. 2002; Williams 2003). This has led to the notional concept of a rotating fluid that is stirred on small scales and within which kinetic energy (KE) cascades to larger scales via nonlinear interactions. The formation of jetlike structures occurs through the effects of anisotropies introduced by the presence of a "planetary vorticity gradient," because of which eddies of a large enough scale are constrained to propagate zonally in a dispersive manner reminiscent of Rossby waves. This results eventually in an accumulation of kinetic energy in zonally elongated structures, ultimately in the form of zonal jets. The mechanism for scale selection of such jetlike structures, however, has remained somewhat obscure, although the scale separating jets that emerge in modeled flows often seems to be comparable with the so-called Rhines scale $L_{R} \simeq$ $\left(U_{\mathrm{rms}} / \beta\right)^{1 / 2}$ (Rhines 1975), where $U_{\mathrm{rms}}$ is a measure of the rms total horizontal velocity fluctuations and $\beta$ is the planetary vorticity gradient $[=(2 \Omega \cos \phi) / a$ for a spherical planet, where $\Omega$ is the planetary rotation rate, $a$ is the planetary radius, and $\phi$ is latitude]. Others (e.g., Galperin et al. 2001; Sukoriansky et al. 2002) have attributed at least part of the scale selection mechanism to the balance between upscale energy transfer and processes removing energy at the largest scales. Under some circumstances (including systems dominated by Ekman bottom friction) this leads to a direct connection between the so-called frictional wavenumber (at which frictional and dynamical time scales are roughly comparable) and the Rhines scale, though for other conditions the scale selection mechanism is less clear (see, e.g., Sukoriansky et al. 2007).

While such models can provide useful and interesting insights into possible mechanisms for banded flows, they are highly idealized and take little account of the vertical structure of realistic geophysical fluid systems. It is sometimes difficult, therefore, to relate results from such studies directly to geophysical problems. Laboratory experiments can provide an alternative means for studying these processes in a real fluid, for which approximations and assumptions underlying simplified models do not apply. In the present context, a $\beta$ effect can be emulated by use of conically sloping topography, at least for quasi-barotropic flow (e.g., Read 2001). However, previous investigations to date (Mason 1975; Condie and Rhines 1994; Bastin and Read 1998) have been unable to access regimes at a sufficiently high Reynolds number (or low enough Ekman number) to be able convincingly to demonstrate fully developed, nonlinear zonation effects.

Here we report the results of new experiments, conducted on the world's largest rotating platform, which seek to confirm that multiple zonal jets may indeed be generated and maintained by internal nonlinear zonation processes. Some preliminary results from this study were published by Read et al. (2004), in which some of the basic flow patterns were discussed and aspects of the anisotropic spectra presented for two of the main experimental cases investigated. In this paper, we present a more detailed analysis of the results of these experiments, considering not only the form of the flow pattern that may develop in the presence of background rotation and sloping or flat bottom topography, but also the detailed interactions between different scales of motion in terms of energy and potential vorticity. Section 2 describes the experimental configuration and introduces the main analysis methods. Section 3 presents an overview of the main scales of motion and 

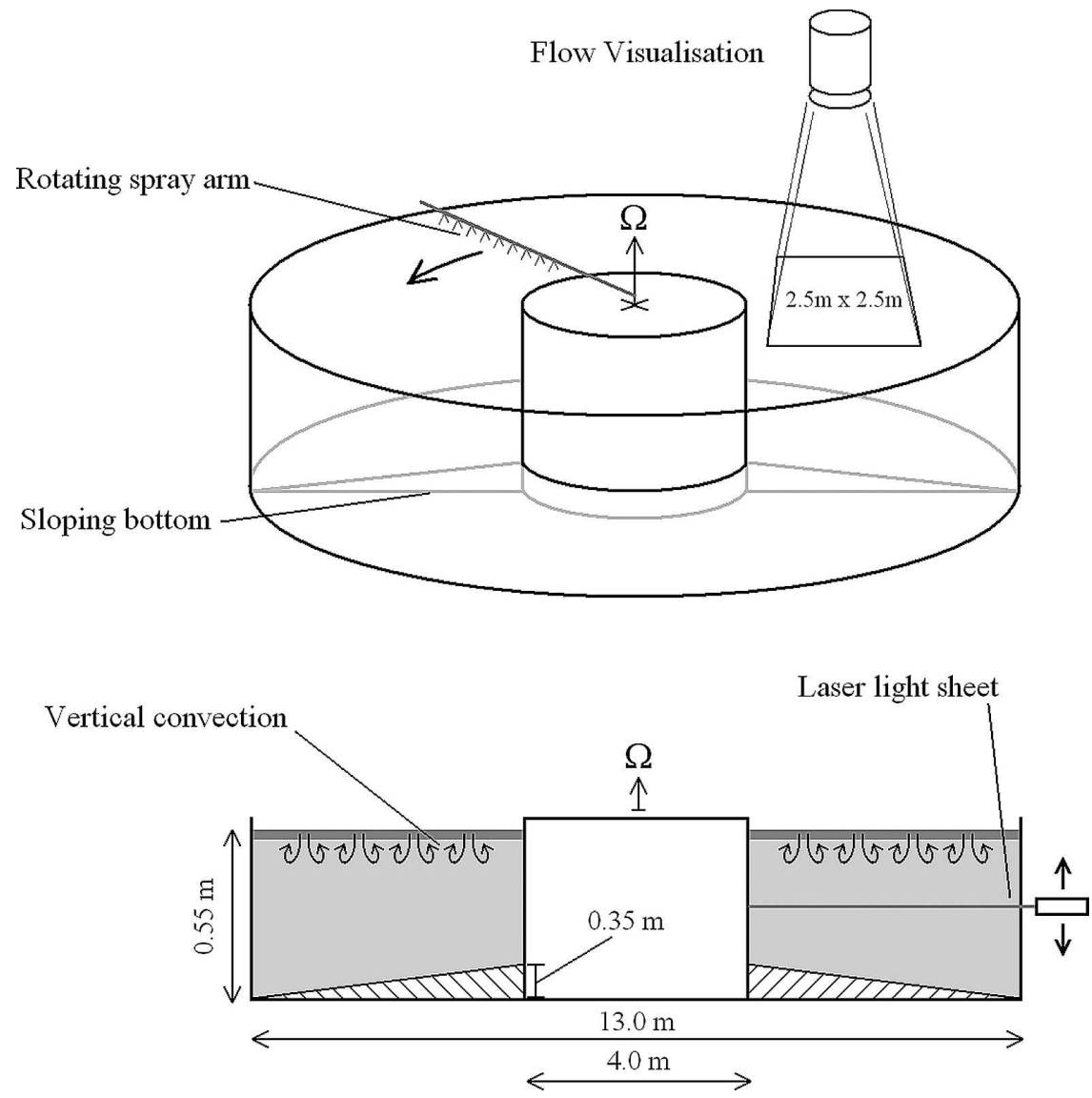

FIG. 1. Schematic cross section of the circular container, showing the rotating spray arm used to provide continuous convective forcing and the field of view of the narrow-angle camera. The entire apparatus is set on a circular turntable.

the range of phenomenology observed during the sequence of experiments. Sections 4 and 5 examine diagnoses first of the direct eddy-zonal flow interactions, and then the detailed two-dimensional kinetic energy spectra and anisotropic transfers in Fourier space. Section 4 considers the observed structure of the flow in terms of potential vorticity dynamics and instabilities. The final section 7 discusses all of these results in the context of the geophysical and planetary systems introduced above.

\section{Apparatus and data analysis}

The experiments were performed on the 13-mdiameter Coriolis rotating platform in Grenoble, France. The schematic layout of the configuration is illustrated in Fig. 1 and the values of the main parameters are listed in Table 1. The experiment consisted of a large circular cylindrical container of diameter $13.0 \mathrm{~m}$ and maximum depth $h=1.5 \mathrm{~m}$ set coaxially on the turntable. An equivalent topographic $\beta$ effect was obtained by imposing a conical slope at the bottom of the circular container, such that the imposed planetary vorticity gradient, $\beta$ was given by

$$
\beta=\frac{2 \Omega}{\bar{h}} \frac{d h(r)}{d r}
$$

where $r$ is the radial coordinate and $\Omega$ the rotation rate of the tank. The bottom slope was implemented by using a plastic-coated fabric sheet, stretched between a circular tubular frame, set just inside the outer radius of the tank, and a circular jig close to the center of the tank whose height could be adjusted via a clamp attached to a post on the axis. This sheet could also be removed relatively easily to enable additional experiments with a flat bottom, in order to compare the flow structure both with and without a topographic $\beta$ effect. The upper surface was free and open to the air. This meant that there was unavoidably a weak centrifugal deformation of the upper surface, even when the bot- 
TABLE 1. Experimental parameters for the three cases I-III covering the full range of $\beta$ obtainable. Here $\tau_{r}$ is the rotation period of the turntable while $\mathrm{T}_{E}$ is the mean Ekman spindown timescale $=h / \sqrt{\nu \Omega}$.

\begin{tabular}{lccccrr}
\hline \hline Case & $\tau_{r}(\mathrm{~s})$ & Bottom slope $\left(^{\circ}\right)$ & $\beta\left(\mathrm{m}^{-1} \mathrm{~s}^{-1}\right)$ & $F_{B}\left(\times 10^{-8} \mathrm{~m}^{2} \mathrm{~s}^{-3}\right)$ & $U_{\mathrm{rms}}\left(\mathrm{cm} \mathrm{s}^{-1}\right)$ & 0.20 \\
I & 40 & 0 & 0.004 & 4.6 & 0.67 & 1387 \\
II & 80 & 4.5 & 0.035 & 21.0 & 0.34 & 1962 \\
III & 40 & 4.5 & 0.08 & 4.5 & 883 \\
\hline
\end{tabular}

tom surface was flat. At the highest rotation rate used, however, this led to a difference in height across the tank from the center to the rim of only $\sim 5 \mathrm{~cm}$. The rotation period of the platform, $\tau_{r}$, was set to $\tau_{r}=40 \mathrm{~s}$ for most experiments, though some additional experiments were carried out with a rotation period of $\tau_{r}=80$ s. The working fluid was a homogeneous weak salt solution of mean density $1026 \mathrm{~kg} \mathrm{~m}^{-3}$, within which small, neutrally buoyant particles (of diameter $0.5 \mathrm{~mm}$ ) could be suspended for flow visualization and measurement (see below).

\section{a. Convective forcing}

A key objective of this study was to investigate the properties of geostrophic turbulence maintained by a mechanism that was as "natural" and unconstrained as possible. In particular, it was considered important to apply forcing on a relatively small horizontal scale, but one that was not fixed in position relative to the rotating reference frame.

To achieve this, convective forcing was applied by gently spraying relatively dense, salty water onto the main fluid layer from a reservoir located above the center of the tank. A system of spray nozzles was set up along a radial arm, which could be rotated around the tank in either direction at a speed controlled independently of the rotation of the main tank. The spacing of the nozzles was carefully designed so that, as the arm was rotated, the flux of salty water deposited onto the free upper surface of the tank was as uniform as possible. In practice, tests indicated that the volume flux from this system was uniform across the entire radius of the tank to better than $\pm 10 \%$. The flow speed and direction of each jet was set so as to disturb the surface of the water as little as possible. For most runs carried out, the spray arm was rotated with a period of 30-50 s in either the prograde or retrograde direction while depositing approximately $5-7 \mathrm{~L} \mathrm{~min}^{-1}$ of salty water.

By varying the density of the water spray a range of buoyancy fluxes $F_{B}=g(\Delta \rho / \rho) F_{V}$ (where $g$ is the acceleration due to gravity, $\Delta \rho$ is the difference in density between the working fluid and spray, and $F_{V}$ is the volume flux of the spray) could be obtained ranging from approximately $10^{-9}$ to $10^{-7} \mathrm{~m}^{2} \mathrm{~s}^{-3}$. For most runs a buoyancy flux of around $F_{B}=4.5 \times 10^{-8} \mathrm{~m}^{2} \mathrm{~s}^{-3}$ was obtained. Together with a rotation rate of (1-2) $\pi / 40$ $\mathrm{rad} \mathrm{s}^{-1}$ and a depth $h$ of around $30-40 \mathrm{~cm}$, this would be expected to lead to a field of convection with a "natural Rossby number," Ro* (Fernando et al. 1991; Maxworthy and Narimousa 1994; Marshall and Schott 1999), defined by

$$
\mathrm{Ro}^{*}=\left(\frac{F_{B}}{f^{3} h^{2}}\right)^{1 / 2},
$$

(where $f=2 \Omega$ ) of around $1-3 \times 10^{-3}$. Such a value would indicate a convective regime somewhat similar to that of deep convection in the terrestrial oceans (Marshall and Schott 1999), and imply that rotation should exert a strong influence on the form of convection.

\section{b. Flow measurement}

The horizontal velocity fields were obtained from plan view images from a charge-coupled device (CCD) camera by a correlation imaging velocimetry (CIV) method (Fincham and Spedding 1997; Fincham and Delerce 2000). Neutrally buoyant tracer particles of diameter $0.5 \mathrm{~mm}$ were suspended in the flow and illuminated by a beam from a 6-W argon ion laser located at the side of the tank and introduced through a glass panel in the outer wall. The beam was rapidly scanned in the horizontal to create a diverging light sheet across a chord through the annular channel, at a depth that could be selected by moving the laser on a computercontrolled carriage.

The illuminated tracer particles were observed from above at a height of approximately $4 \mathrm{~m}$ above the surface of the tank by two digital cameras, a lowresolution, wide-angle camera with a resolution of $768 \times 484$ and covering an area $4.5 \mathrm{~m} \times 3.3 \mathrm{~m}$, and a higher-resolution, narrow-angle camera with a resolution of $1024 \times 1024$ and covering an area $2.5 \mathrm{~m} \times 2.5 \mathrm{~m}$. This allowed the observation of the largest feasible area of the tank, given the limited height of the building in which the rotating facility was accommodated, though it represented a maximum of just $12 \%$ of the total surface area of the annular channel that was visible simultaneously. By aligning the long axis of the wide-angle camera with the radius of the tank, however, the opti- 
mum coverage of the zonal flow profile across the tank could be obtained. This did mean that a true instantaneous zonal average could not be measured directly, though use of time averaging would help to recover a more global sample of flow statistics.

Flow fields were obtained every $20 \mathrm{~s}$ from groups of images obtained by each camera over time intervals of up to $3 \mathrm{~s}$. By applying CIV analysis to these bursts of images, velocities could be measured to a precision of better than $\pm 0.1 \mathrm{~mm} \mathrm{~s}^{-1}$ (relative to typical velocities of a few millimeters per second) on a $72 \times 48$ point (wide angle) or $98 \times 98$ point (narrow angle) rectangular grid. To diagnose the azimuthal anisotropy of the evolving flow, the velocity fields were subsequently decomposed into radial and zonal (i.e., azimuthal) components and mapped into $(r, r \theta)$ coordinates, where $r$ is the radius from the center of the tank and $\theta$ the azimuthal angle (increasing counterclockwise from above; see Fig. 2a). For the purposes of presentation and analysis, we also make use of a simple local Cartesian system with $x$ in the (clockwise) $\theta$ direction (so $x=-r \theta$, usually measured from the radial center line of the camera image) and $r$ in the outwards radial direction.

In addition to long series of horizontal flow fields, a few measurements were made of the flow in the azimuth-height $[(r \theta, z)$ or $(-x, z)]$ plane near midradius by reorienting the laser light sheet into a vertical plane and viewing with the wide-angle camera through a second glass panel in the outer wall. This enabled the flow over a $0.5 \mathrm{~m} \times 0.5 \mathrm{~m}$ area to be measured in order to examine the flow pattern forced directly by the overhead spray.

The vertical profile of density in the flow could be measured at intervals to an accuracy of approximately $\pm 1 \mathrm{~kg} \mathrm{~m}^{-3}$ using a conductivity probe that was mounted on the operator gantry at midradius and could be driven downward into the flow from the surface to within $10 \mathrm{~cm}$ of the bottom of the tank on command via a motor-driven carriage.

\section{Scales and flow morphology and variability}

In this section we describe the basic flow structures that were obtained over a range of conditions. Because of the limited time available for this investigation, only a few combinations of parameters could be explored. Accordingly we concentrate on discussing a set of just three runs whose parameters are listed below in Table 1. A few additional short experiments were also carried out at other parameter settings (especially with varying buoyancy forcing), though with only limited flow measurements.

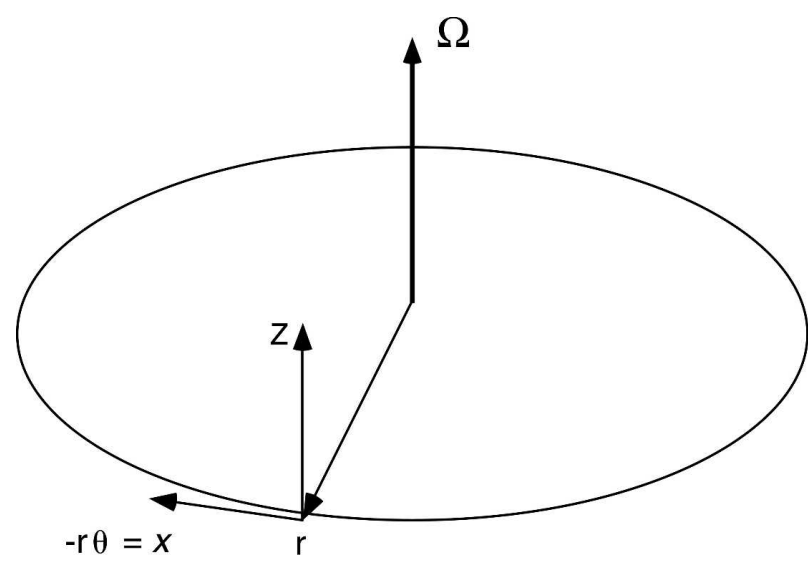

FIG. 2. The coordinate systems used in this paper in relation to the cylindrical tank. For some purposes, the cylindrical coordinate system $(r \theta, z)$ is used alternately with a local Cartesian system $(x$, $r, z)$, for which $x=-r \theta$ is in the clockwise $(-\theta)$ direction.

\section{a. Vertical measurements}

As discussed above, the forcing of the flow was effected by spraying dense, salty water onto the free surface of the tank from a rotating arm in the frame of the rotating table. This initially produced a shallow layer of dense fluid on the top surface of the flow that rapidly became statically unstable (on a time scale of a few seconds), leading to the formation of discrete plumes of salty water that would then descend toward the bottom of the tank. This is illustrated in Fig. 3, which shows two dye visualizations of descending plumes in the azimuthheight $[(r \theta, z)$ or $(-x, z)]$ plane of the tank close to midradius for a case with a flat bottom, a rotation period of $80 \mathrm{~s}$ and buoyancy flux of approximately $1.7 \times$ $10^{-7} \mathrm{~m}^{2} \mathrm{~s}^{-3}$.

Fluorescein dye was introduced at the surface of the tank as the spray arm moved overhead above the field of view. This was then entrained into the plumes, which could be seen descending and spreading horizontally as they moved toward the bottom of the tank. It is noteworthy from such images (e.g., in Fig. 3b) that, although the convective mixing is initially most vigorous in the uppermost $25 \%$ of the depth of the tank (Fig. 3a), individual plumes actually reach the bottom of the tank, albeit with some azimuthal tilting, indicative of deep, penetrative convection.

The velocity field associated with these plumes was obtained via CIV analysis of sequences of these images, using the dispersion of neutrally buoyant particles to trace the flow. A typical example of such a velocity field is shown in the $(x, z)$ plane at midradius in Fig. 4 .

The velocity field is evidently dominated by irregular descending jets of fluid associated with each plume, on a horizontal scale of around $10 \mathrm{~cm}$, again clearly tra- 
(a)

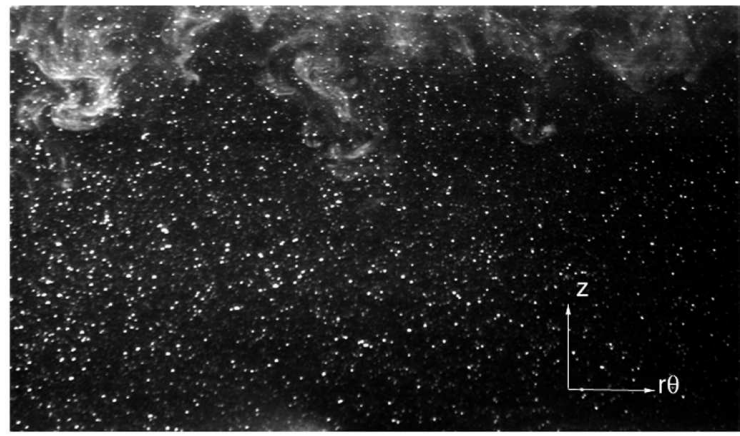

(b)

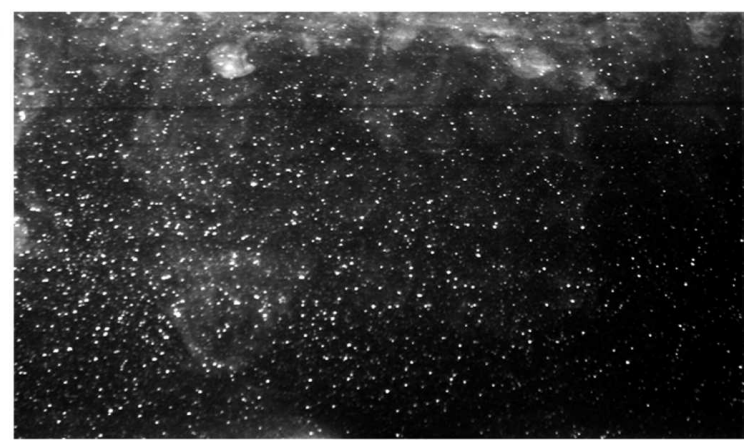

FIG. 3. Two visualizations of convective plumes in the azimuthheight plane of the Coriolis tank, rotating with a period of $80 \mathrm{~s}$ and with a flat bottom and viewed from the side using a wide-angle digital camera. The field of view is approximately $0.74 \mathrm{~m} \times 0.55 \mathrm{~m}$ in $(r \theta, z)$, with the bottom of the tank approximately at $z=0$, and plumes are visualized using fluorescein dye introduced at the top surface as the spray arm moves overhead across the field of view. The two images are separated by approximately $110 \mathrm{~s}(\sim 1.5$ rotation periods).

versing the entire depth of the tank along a sloping trajectory. The flow pattern appears to evolve rapidly, on time scales less than the rotation period of $80 \mathrm{~s}$, thus representing a significantly ageostrophic, three-dimensional convective flow on these scales.

Although this convective flow would suggest the presence of a statically unstable stratification, in practice the conductivity probe always measured either a neutral or weakly stable density gradient. The vertical density gradient was typically found to evolve during the course of an experiment lasting a few hours, beginning with essentially neutral stratification and then gradually developing a weakly stable gradient in the lower part of the tank with a maximum value of $\partial \rho / \partial z$ of around $1-2 \mathrm{~kg} \mathrm{~m}^{-4}$. The upper part of the tank remained approximately neutrally stratified with no clear evidence of unstable stratification, which was probably confined to a thin layer near the top surface, though this could not be measured reliably.

These measurements would suggest a crude estimate of an upper limit on $N^{2}$ of $\sim 5 \times 10^{-3} \mathrm{~s}^{-2}$, where

$$
N^{2}=-\frac{g}{\rho_{0}} \frac{\partial \rho}{\partial z},
$$

$\rho$ is the fluid density (with $\rho_{0}$ a reference value), and $g$ is the acceleration due to gravity. This would also imply a corresponding lower limit on the internal deformation wavenumber,

$$
k_{d}=f /(N h),
$$

where $k_{d} \geq 13 \mathrm{rad} \mathrm{m}^{-1}$ for cases I and III and $k_{d} \geq 6.5$ $\operatorname{rad~} \mathrm{m}^{-1}$ for case II.

\section{b. Horizontal flow patterns}

For each main experiment, the fluid in the tank was brought into solid-body rotation with the tank over a period of at least half a day prior to the start of the experiment, without any form of forcing. For the majority of experiments carried out, horizontal velocity fields were derived at 20-s intervals, beginning shortly after the initiation of convective forcing, and continued for as long as possible. In at least two cases, the flow continued to be monitored some time after the convective forcing was removed, thereby providing information on the decay of the turbulence field, due mainly to Ekman friction. The horizontal flow was typically measured at a level close to middepth (approximately $25 \mathrm{~cm}$ below the top surface in each case), although in at least two runs (with a flat bottom) a short sequence of measurements were made in which the horizontal flow field was sampled at a series of five different levels spanning the full depth of the tank.

\section{1) Flat BOTTOM Flows}

The first case we consider is for a flat bottom, in which the fabric sheet was loosened sufficiently to lie at the bottom of the tank. In this case there is only a weak topographic gradient across the tank, due solely to the centrifugal distortion of the free upper surface. In practice at a rotation period $\tau_{r}=40 \mathrm{~s}$ this led to a mean slope in the top surface of around $0.65^{\circ}$ and a corresponding value for $\beta$ [cf. Eq. (1)] of $\sim 0.0036 \mathrm{~m}^{-1} \mathrm{~s}^{-1}$. Although this case corresponds most closely to an $f$ plane, therefore, there is a weak residual $\beta$ effect that should not be ignored. However, the effect is comparatively weak, and leads to characteristic length scales based on $\beta$ [such as the Rhines scale

$$
L_{R}=\pi\left(U_{\mathrm{rms}} / \beta\right)^{1 / 2},
$$

where $U_{\text {rms }}$ is a typical horizontal velocity scale based on the root-mean-square velocity fluctuations] that are comparable with the size of the tank. For run I, for 


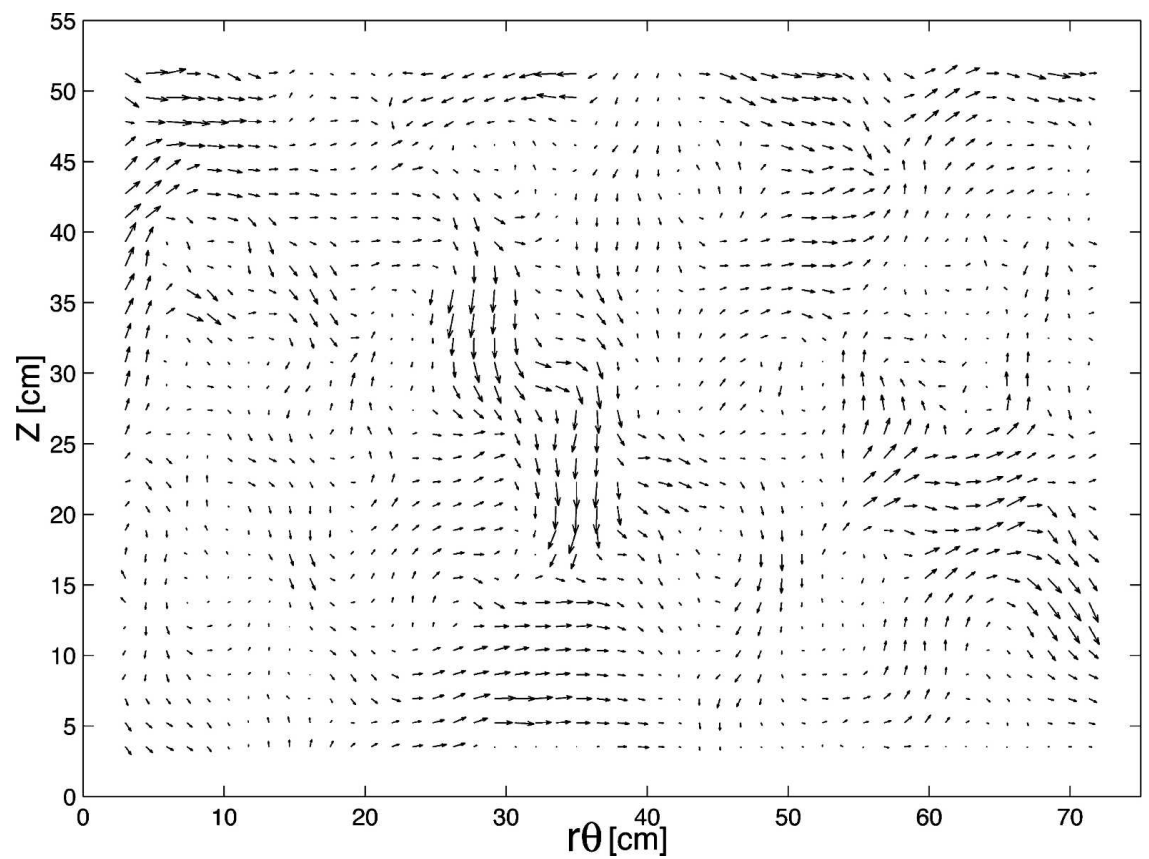

FIG. 4. Flow field in the presence of convective plumes in the azimuth-height plane of the Coriolis tank, rotating with a period of $80 \mathrm{~s}$ and with a flat bottom and viewed from the side using a wide-angle digital camera. The maximum vector length corresponds to a velocity of approximately $10 \mathrm{~mm} \mathrm{~s}^{-1}$.

example, $L_{R} \simeq 2 \mathrm{~m}$, indicating a Rhines wavelength of around $4 \mathrm{~m}$.

Figure 5 shows a typical example of an equilibrated horizontal flow, taken from run I using the wide-angle camera. In this case, the flow is apparently dominated by a single complex anticyclonic gyre, around $2 \mathrm{~m}$ across in radius though perhaps more extended in azimuth beyond the field of view. In addition, there is a general tendency for broadly retrograde drift in the azimuthal direction toward the outer radius and prograde drift in the inner part of the tank. This trend is clearly seen in the azimuthal mean azimuthal velocity profile in the left frame of Fig. 5. This overall pattern was seen to persist for long periods of the experiment, with the anticyclonic gyre remaining almost stationary, though evolving erratically in shape and intensity.

It was subsequently noted that the location of the inclined prograde jet on the inner flank of the gyre (at around $r=0.5 \mathrm{~m}$ and $x=0$ ) coincided with some folds and irregularities in the fabric sheet that led to some residual topographic ridges $1-2 \mathrm{~cm}$ in height. It was possible, therefore, that some weak topographic effects were present to render the main flow pattern nearly stationary relative to the bottom of the tank. The weak differential azimuthal drift with radius is also in the same sense as might be expected from the residual effects of wind stress or drag, produced by differential motion between the moving surface of the tank and the stationary air in the laboratory, though it was difficult to estimate this effect theoretically. Nevertheless, the influence of these experimental imperfections needs to be borne in mind in interpreting these results.

\section{2) Sloping BotTom Flows}

In cases designated as having a sloping bottom, the fabric sheet was placed under tension between the tubular steel frame along the outer radius and a circular harness attached to the central post of the tank. This enabled a conically sloping surface to be obtained, once the fluid in the tank had spun up to solid-body rotation, with a slope angle of approximately $5^{\circ}$, decreasing the depth of the tank to around $25 \mathrm{~cm}$ at the inner radius of the annular channel (at $r=2.5 \mathrm{~m}$ ). Several cases were run in this configuration, varying the buoyancy flux (by changing the density difference between the tank fluid and the spray) over around an order of magnitude. Good statistics, however, were only measured for a few cases owing to difficulties with flow visualization and control of the spray forcing during the early phases of the experimental investigation.

For illustration, we present here results from a long duration experiment (case III, lasting some $6 \mathrm{~h}$ ) corresponding to a mean buoyancy flux of $4.5 \times 10^{-8} \mathrm{~m}^{2} \mathrm{~s}^{-3}$ 


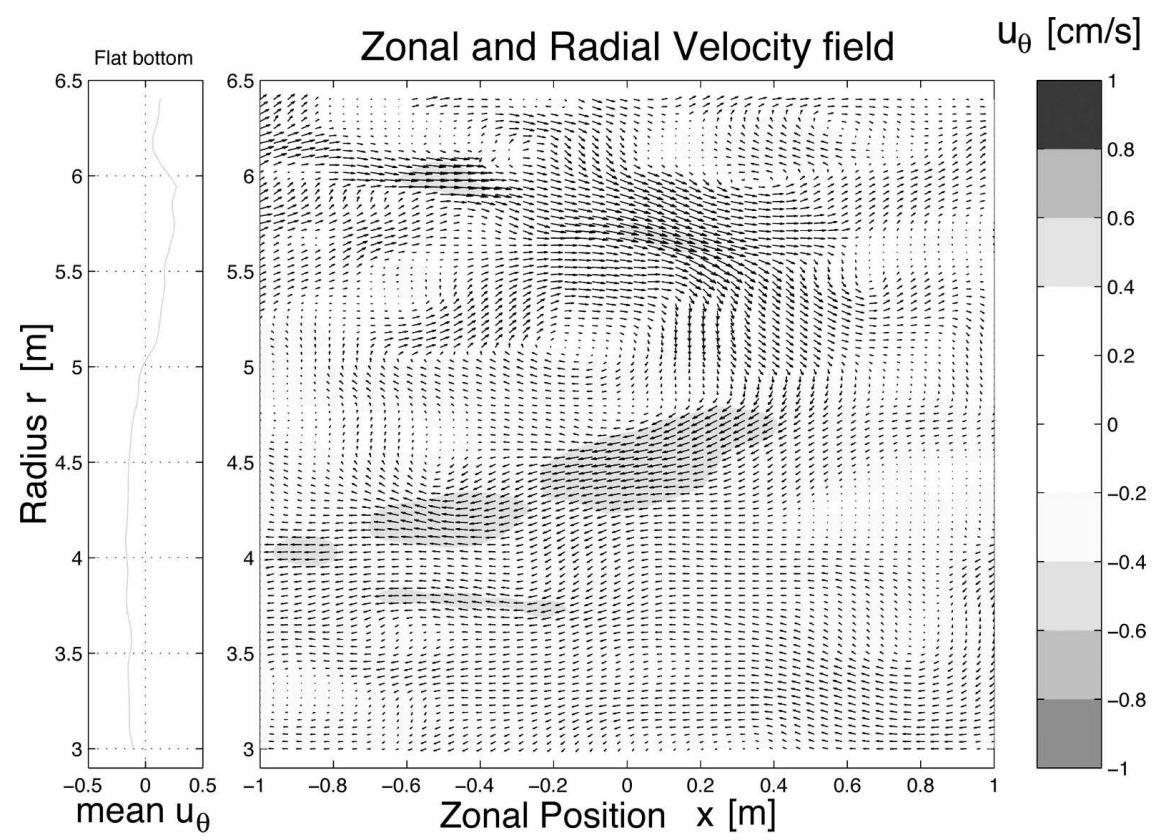

FIG. 5. Horizontal flow field at midheight in the Coriolis tank, rotating with a period of 40 $\mathrm{s}$ and with a flat bottom. Shaded contours are of azimuthal velocity $u_{\theta}\left(\mathrm{cm} \mathrm{s}^{-1}\right)$. The maximum vector length corresponds to a velocity of approximately $0.6 \mathrm{~cm} \mathrm{~s}^{-1}$. The azimuthal average profile of $u_{\theta}$ is shown in the left-hand frame.

(i.e., comparable to the case I, discussed above), and with a rotation period of $40 \mathrm{~s}$.

The appearance of the flow in this case is quite different to case I discussed above. Overall the flow is dominated by a mixture of small gyrelike eddies of typi- cal diameter $0.5-1 \mathrm{~m}$ interspersed with meandering, azimuthally oriented currents. The latter also appear clearly in the azimuthal mean azimuthal flow profile in the leftmost frame of Fig. 6, in which "jets" of alternating sign are found with peak amplitudes of up to 0.5

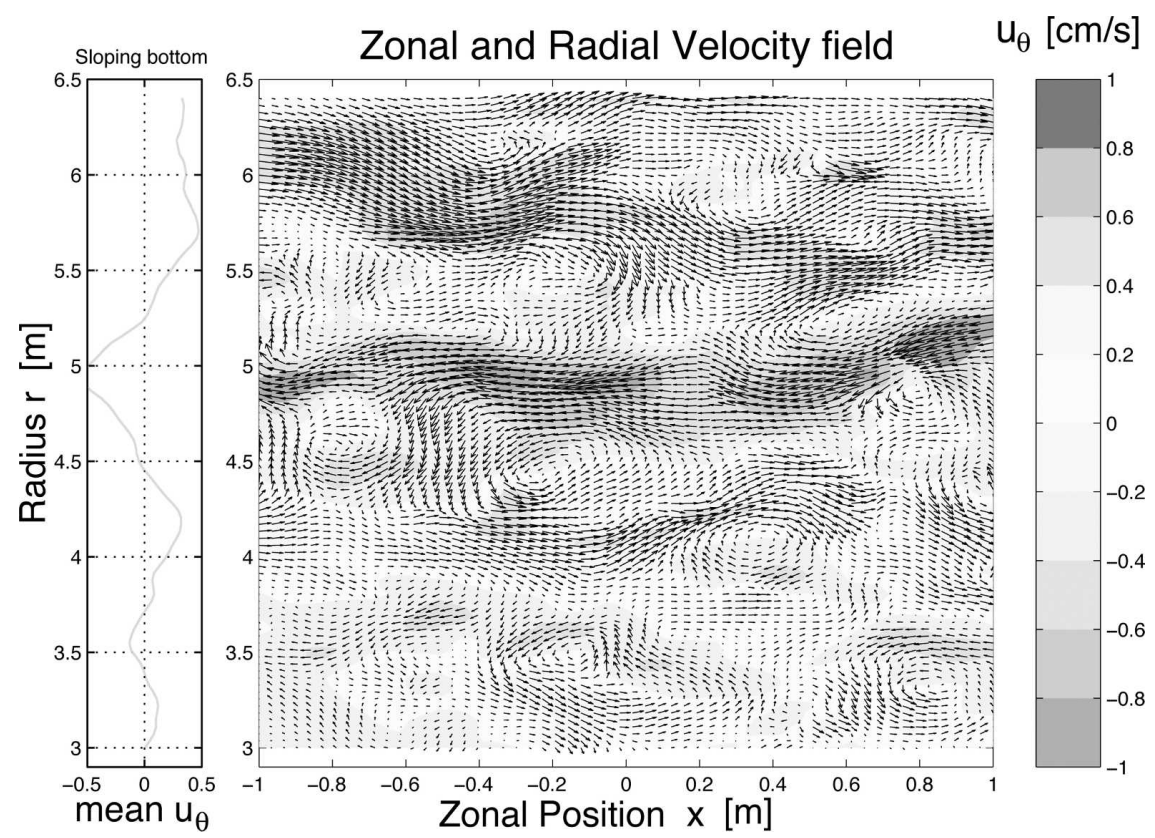

FIG. 6. Same as in Fig. 5, but for a sloping bottom. 
(a)

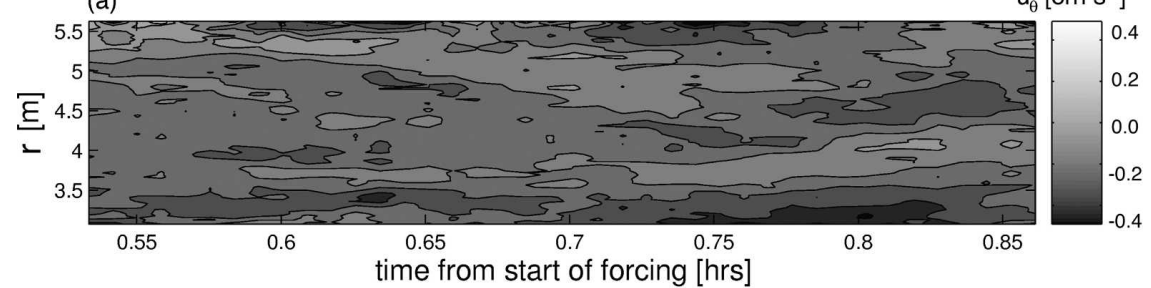

(b)
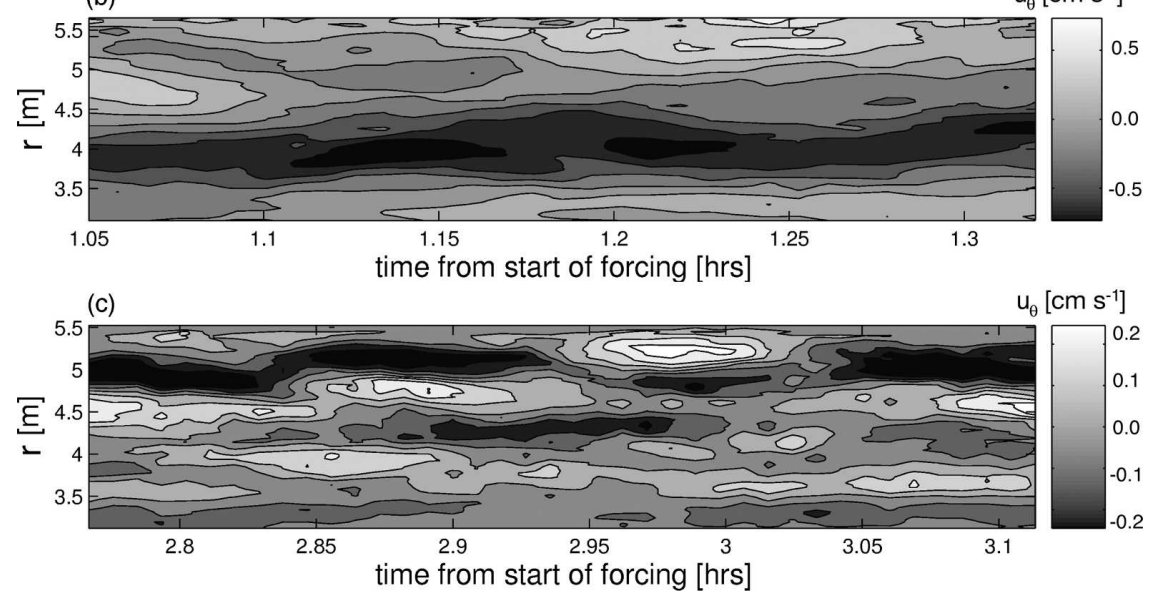

FIG. 7. Azimuth-time contour plots of azimuthal mean flow at midheight in the Coriolis tank, for the three main cases discussed in the text: (a) I (rotation period $40 \mathrm{~s}$, flat bottom), (b) II (rotation period $80 \mathrm{~s}$, sloping bottom), and (c) III (rotation period $40 \mathrm{~s}$, sloping bottom). Shaded contours are of azimuthal velocity $\left(\mathrm{cm} \mathrm{s}^{-1}\right)$.

$\mathrm{cm} \mathrm{s}^{-1}$. This banded organization of the zonal flow is also apparent in the shading of Fig. 6, in which clear stripes or bands oriented azimuthally are clearly seen. Flow near the outer boundary, however, is predominantly retrograde, much as for case I in Fig. 5.

Eddy gyres of either sign are apparent, though they typically are found at radii in which the vorticity of the azimuthal currents are of the same sign as the gyres. As discussed below, unlike in case I, these gyres continually evolve and, like other meanders in the azimuthal jets, translate azimuthally across the measurement domain, moving predominantly in the retrograde direction. Individual eddy gyres typically persisted for longer than the transit time across the field of view and formed reasonably coherent structures. They had a typical horizontal aspect ratio not far from unity, though their shape and orientation were found to evolve chaotically as they moved across the field of view.

\section{3) TIME VARIATIONS OF JETS}

As discussed above, the bottom slope was found to have a strong influence on the time evolution of the nonaxisymmetric eddies and gyres, with a tendency for stationary, nonpropagating structures with a flat bottom and retrograde-propagating eddies with a sloping bottom. Such behavior is suggestive of the eddies acting in a manner similar to Rossby waves, which propagate in a retrograde sense at a speed proportional to $\beta$. In the present case III, large-scale eddies with an azimuthal wavelength of around $1 \mathrm{~m}$ were seen to propagate azimuthally at a speed of around $2 \mathrm{~mm} \mathrm{~s}^{-1}$ across the domain at most radii.

During this time interval, however, the zonal flow pattern was also observed to evolve, although the basic qualitative character of the flows shown above were preserved.

In particular, cases with a sloping bottom were found to produce fields of relatively small vortices in association with an azimuthal mean flow with increasing amounts of structure and variability as the topographic $\beta$ was increased. Such a trend is clearly shown in Fig. 7, which presents radius-time contour maps of azimuthal mean azimuthal velocity across the field of view of the narrow-angle camera. From these maps, it is clear that the flow breaks up into patterns of meandering zonal jets as $\beta$ is increased. The position and amplitude of each jet is not constant, however, as apparent in Fig. 8, which shows a sequence from the strongest $\beta$ case III over a longer time interval. But the flow evidently evolves erratically with time, with individual jets ap- 


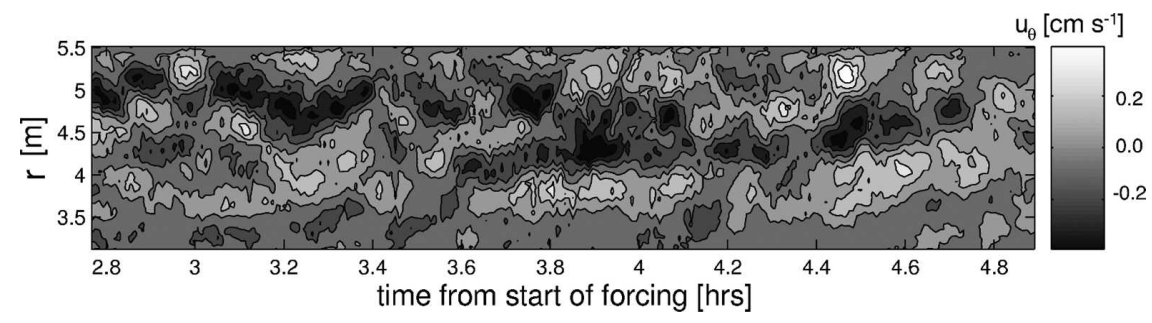

FIG. 8. Azimuth-time contour plot of mean azimuthal flow at midheight in the Coriolis tank, for case III (rotation period $40 \mathrm{~s}$, sloping bottom) over a period of approximately $2 \mathrm{~h}$. Shaded contours are of azimuthal velocity $\left(\mathrm{cm} \mathrm{s}^{-1}\right)$.

pearing to split and merge. The total number of jets, however, remains more or less the same throughout the period of observation, though it may be that some slow, systematic evolution is apparent. This might suggest that the jets are emerging during a long transient phase, even though the average kinetic energy of the flow reaches an equilibrium level within 1-2 $\mathrm{h}$ of turning on the forcing. Such a possibility is discussed below in the context of other model studies.

\section{c. Vertical flow structure}

Although the basic buoyancy-driven forcing of the flows was essentially baroclinic in character, various theoretical and modeling studies suggest (Salmon 1980) that the evolution of any upscale nonlinear energy cascade will lead to the excitation of large-scale flow components of an essentially barotropic character. Although time did not permit an extensive study of this aspect of the flow evolution, a few near-simultaneous measurements were made of the horizontal flow field at several levels in the vertical for one case with a flat bottom.

The flow was allowed to develop for several hours while buoyancy forcing was maintained at a constant value of around $F_{0} \simeq 4 \times 10^{-8} \mathrm{~m}^{2} \mathrm{~s}^{-3}$. Figure 9 shows results from the near-simultaneous measurement of horizontal velocity at five vertical levels at a particular instant, spanning almost the full depth of the tank. Figure 9a shows shaded contours of the vertically averaged flow, while Fig. 9b shows a map of the standard deviation of KE about that vertical mean. Thus, Fig. 9a may be regarded as representing the $\mathrm{KE}$ of the barotropic component of the flow, while Fig. $9 \mathrm{~b}$ represents a measure of the baroclinic velocity field.

From these maps, it is clear that the vertically averaged (barotropic) flow contains much more kinetic energy than the standard deviation (baroclinic) components, by a factor of 10 in peak amplitude (note the difference in contour scale between Figs. 9a and 9b). In addition, the vertically averaged flow appears to be dominated by relatively large-scale structures, while the baroclinic standard deviation field is dominated by small-scale patches of KE, with a typical diameter of around $10-20 \mathrm{~cm}$. Such a partitioning of scale and magnitude between the barotropic and baroclinic components of the flow is quite striking, and appears to demonstrate strongly the trends suggested from simple model studies in forming a strongly barotropic largescale flow from a baroclinic (convective) energy input.

The typical scale of the baroclinic features presumably represents the horizontal size of individual convective plumes in the flow, with reference to Figs. 3 and 4 above. It is of interest that this scale is comparable to the scale $l_{\text {rot }}$, defined by

$$
l_{\text {rot }} \sim(3-5)\left(\mathrm{Ro}^{*}\right)^{1 / 2} h,
$$

and suggested by Fernando et al. (1991) and Marshall and Schott (1999) as a likely horizontal scale for rotationally dominated convective plumes. Given the value of natural Rossby number Ro* $\sim 3 \times 10^{-3}$ and $h \simeq$ $30-40 \mathrm{~cm}$, this would be expected to lead to $l_{\text {rot }} \sim 10-20$ $\mathrm{cm}$, much as observed here in Fig. 9b. Moreover, the observed velocity fluctuations in $u_{\theta}$ and $w$ on the scale of the plumes is $O\left(1 \mathrm{~cm} \mathrm{~s}^{-1}\right)$, again consistent with the scalings suggested by Fernando et al. (1991) and Marshall and Schott (1999) of $|u, w| \sim \Omega l_{\text {rot }} \sim 1-2 \mathrm{~cm} \mathrm{~s}^{-1}$.

\section{Eddy-zonal flow interactions}

In considering the origin of the large-scale barotropic flow discussed above, it was suggested that a form of upscale nonlinear cascade of energy could be the mechanism responsible. In effect, this implies that the pattern of slowly varying, barotropic zonal jets arise through direct interactions between nonaxisymmetric eddies and the azimuthally symmetric component of the flow. Such a possibility was effectively proposed for Jupiter's zonal jets by Ingersoll et al. (1981), who presented evidence from analyses of cloud-tracked winds derived from images from the Voyager 1 and 2 space- 

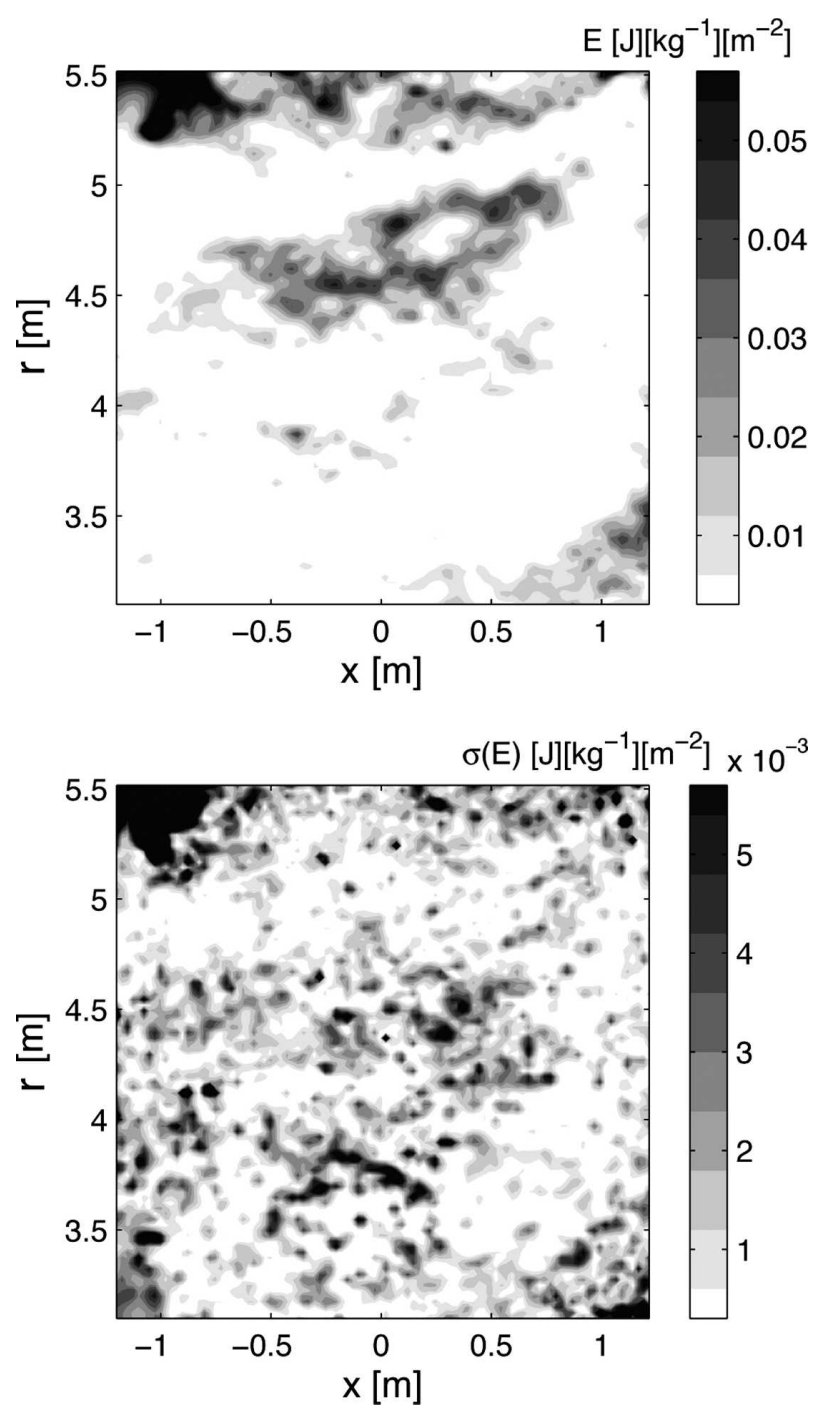

FIG. 9. Instantaneous horizontal kinetic fields in the Coriolis tank, rotating with a period of $40 \mathrm{~s}$ and with a flat bottom. Shaded contours are of kinetic energy per unit mass $\left(\mathrm{J} \mathrm{kg}^{-1} \mathrm{~m}^{-2}\right)$ and show (a) the kinetic energy of the vertically averaged flow and (b) the std dev of kinetic energy about the vertical mean.

craft. The evidence for this mechanism was later questioned by Sromovsky et al. (1982) because of possible biases caused by the nonuniform distribution of cloud tracers obtained by manual selection, though this result has subsequently been confirmed from Cassini data by Salyk et al. (2006). Read (1986) also raised additional doubts over the interpretation of this result, (a) because it was based on an unverified assumption that barotropic motions dominated the flow in the Jovian troposphere, and (b) because the evidence was based on the correlation of the horizontal Reynolds stress component $\overline{u^{\prime} v^{\prime}}$ (where $u$ is the zonal velocity component and $v$ the northward velocity, and primes indicate depar- tures from the zonal mean) with the lateral gradient of zonal flow $\partial \bar{u} / \partial y$, which provides a fundamentally nonlocal measure of zonal flow interaction. Moreover, various other modeling studies appeared to suggest that large-scale nonzonal eddies might derive their energy from barotropic instability of the pattern of zonal jets, thereby linking this with the observed violations of the Rayleigh-Kuo stability criterion in the measured zonal jets around Jupiter's cloud tops.

\section{Reynolds stress accelerations}

Similar issues must surely arise in the present experiments, though in this case we have already established that the large-scale flow (including, presumably, the main eddies resolvable in our measurements) is predominantly barotropic. Given detailed, accurate, and representative measurements of horizontal velocity fields, well sampled in time and covering an area of the flow sufficient to obtain meaningful statistics, it is feasible directly to derive not only the pattern of horizontal Reynolds stress at each time step for which velocities are measured, but also the divergence of this Reynolds stress and hence the implied eddy-induced acceleration of the azimuthal mean zonal flow. In cylindrical geometry (see, e.g., Pfeffer et al. 1974; Read 1985), the full azimuthally averaged zonal momentum equation reduces to

$$
\begin{aligned}
& \partial \bar{u} / \partial t=(2 \Omega+\bar{u} / r) \bar{v}-\bar{v} \partial \bar{u} / \partial r-\bar{w} \partial \bar{u} / \partial z-\frac{1}{r} \partial / \partial r\left(r \overline{u^{\prime} v^{\prime}}\right) \\
&+\partial / \partial z\left(\overline{w^{\prime} u^{\prime}}\right)+\overline{F^{(x)}} \\
& {[6] }
\end{aligned}
$$

[cf. Andrews et al. 1987, their Eq. (3.3.3)], where $u, v$, and $w$ are now the azimuthal, radial, and vertical velocity components, respectively. Here $F^{(x)}$ represents residual forces, for example, due to molecular viscosity and/or wind stresses. Term [2] represents the combined Coriolis and centrifugal acceleration due to zonal mean meridional flow, while [3] and [4] represent advection of zonal momentum by $\bar{v}$. Terms [5] and [6] represent accelerations due to the divergence of the Reynolds stresses. If the present experiment develops zonal jets via the same barotropic nonlinear zonation mechanism as is believed to be responsible for the development of zonal jets in the numerical simulation studies discussed above, the zonal acceleration [1] should be dominated by the effects of term [5] representing the divergence of the horizontal Reynolds stress. Moreover, this balance should be realized independently of height $z$. Thus, if the zonal flow is indeed a direct result of barotropic eddy-zonal flow interactions, it should be possible to compare independent measurements of both the hori- 

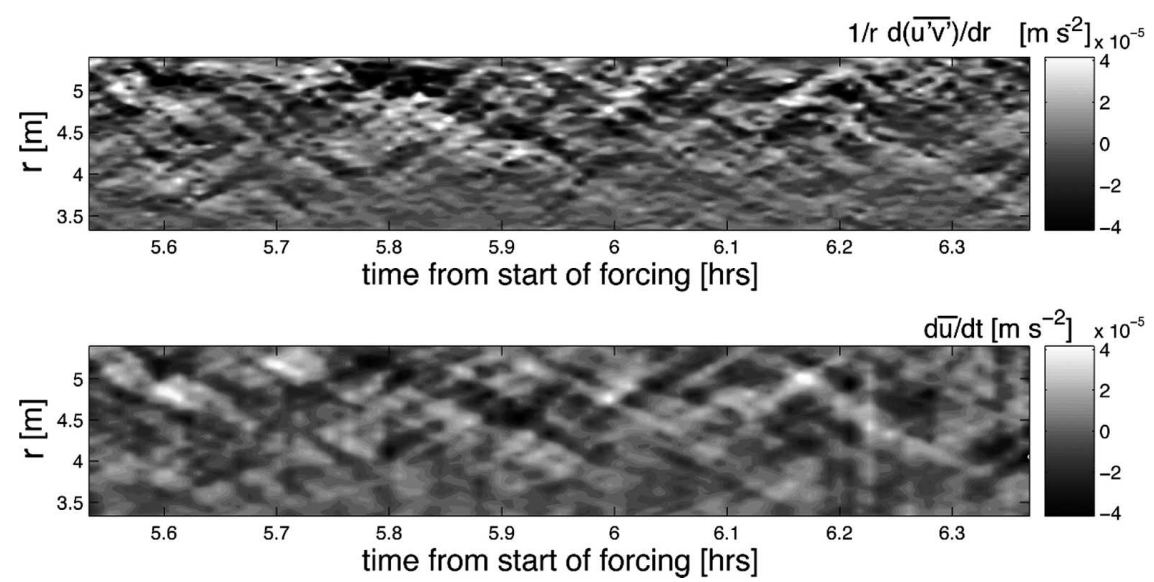

FIG. 10. Measurements of the divergence of the horizontal Reynolds stress (a) in an equilibrated convectively driven flow with a sloping bottom (case III), compared with (b) the measured acceleration $\partial \bar{u} / \partial t$ of the mean azimuthal flow, as determined by finite time differencing. Measurements cover a period of approximately $1 \mathrm{~h}$, sampled twice per rotation period.

zontal Reynolds stress divergence and actual changes in the azimuthal mean flow at any height in the flow.

In Fig. 10 the results of such a comparison are shown, for the case III with the most highly developed pattern of zonal jets featured above. In Fig. 10a, shaded contours of the instantaneous divergence of the measured Reynolds stress are shown, sampled twice per rotation period for a total interval of around $1 \mathrm{~h}$. By this time the forcing had been applied continuously for several hours and the flow seemed to have reached a reasonably equilibrated level of kinetic energy. In Fig. 10b, measurements are shown of the acceleration of the azimuthal mean zonal flow $\partial \bar{u} / \partial t$, obtained by differencing the profile of azimuthal mean flow in successive time steps using centered differences. Both fields appear to be quite noisy, with many patches of both positive and negative accelerations apparent in each field, apparently fluctuating back and forth and mostly concentrated in the outer half of the annular channel. Even so, there are some similarities apparent in the spatial variations of both fields, suggesting that they could be correlated.

Figure 11 shows the result of forming the correlation coefficient $C(r, \tau)$, computed as a function of radius and averaged over all time steps in the narrow-angle dataset for case III, between the two quantities plotted in Fig. 10. The definition of $C$ is given by Eq. (8),

$$
C(r, \tau)=\frac{\left\langle\bar{u}_{t}(r, t+\tau)\left\{\operatorname{div}\left[\overline{u^{\prime} v^{\prime}}(r, t)\right]\right\}\right\rangle}{\left\{\left\langle\left[\bar{u}_{t}(r, t)\right]^{2}\right\rangle\left\langle\left\{\operatorname{div}\left[\overline{u^{\prime} v^{\prime}}(r, t)\right]\right\}^{2}>\right\rangle\right\}^{1 / 2}},
$$

where angle brackets denote the time average, the subscript $t$ denotes time derivative, and $\operatorname{div}()=(1 / r) \partial r() /$ $\partial r$, and allows for the possibility of a time lag in the response between the Reynolds stress divergence and the azimuthal mean zonal flow itself. The results show a clearly peaked response in correlation close to lag zero $(\tau=0)$ and of magnitude up to 0.5 , though with a tendency for the acceleration to respond slightly after the measured Reynolds stress event. There is a clear cutoff on the negative lag (time lead) side of the origin at all radii, with a more gradual decay in correlation with time lag in the positive lag direction. This is a clear signature that the horizontal Reynolds stress is strongly correlated with the measured zonal flow acceleration,

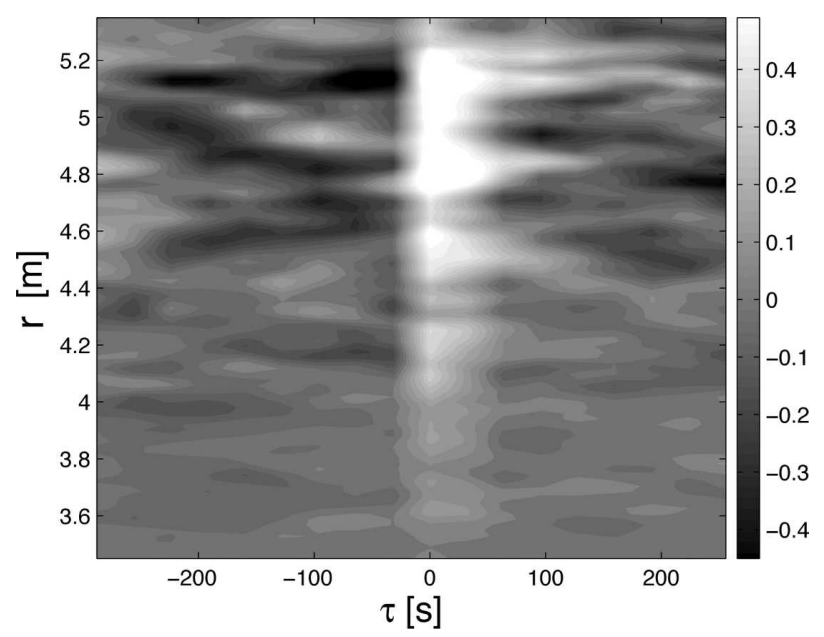

FIG. 11. Shaded contour map of the correlation coefficient between the horizontal Reynolds stress divergence and the zonal flow acceleration in an equilibrated convectively driven flow with a sloping bottom (case III). Covariance is plotted as a function of radius and time lag between the measurement of Reynolds stress and acceleration. Measurements are averaged over a period of approximately $1 \mathrm{~h}$, sampled twice per rotation period. 
and is entirely consistent with the hypothesis that horizontal Reynolds stress plays a major role in controlling and influencing subsequent changes in the behavior of the pattern of azimuthal mean flow. However, it is somewhat surprising that the zonal acceleration takes place following a short time delay. The explanation for this is not clear in the present work, though may indicate a finite time interval needed for disturbances detected at the midplane to propagate their influence throughout the water column.

The magnitude of the implied conversion of kinetic energy from eddies into the zonal flow, corresponding to $-\bar{u} / r \partial\left(\overline{r u^{\prime} v^{\prime}}\right) \partial r$ integrated over the domain, is around $3.0 \times 10^{-8} \mathrm{~W} \mathrm{~kg}^{-1}$, which may be compared with the computed value for the rate of change of zonal kinetic energy $\partial\left(u^{2} / 2\right) / \partial t$ of $3.2 \times 10^{-8} \mathrm{~W} \mathrm{~kg}^{-1}$ for case III. Thus, the Reynolds stresses can account for more than $90 \%$ of the energy fluctuations in the zonal flow for this case. The missing remainder may represent the effects of measurement errors, but could also reflect other sources of zonal acceleration [e.g., due to vertical Reynolds stresses associated with baroclinic (convective) exchanges on small scales], and also the direct effects of wind stresses. The latter are almost certainly dominant for case I, for which Reynolds stresses are too weak to account for observed accelerations.

\section{Kinetic energy spectra and energy transfers}

Numerical studies of small-scale forced twodimensional turbulent flows on the sphere or $\beta$ plane that develop systems of parallel zonal jet streams are often found to develop strongly anisotropic kinetic energy spectra with various characteristic properties. Given the importance of inertial upscale energy cascades in such flows, the form of the energy spectra may be diagnostic of various important aspects of the turbulent dynamics shaping the equilibrated flow. In particular, the logarithmic slope of the energy spectrum may indicate the nature of energy transfers between scales in different directions. But for equilibrated spectra with a constant kinetic energy injection rate $\epsilon$ (in units of $\mathrm{m}^{2} \mathrm{~s}^{-3}$ ), introduced at small scales, the upscale energy-cascading kinetic energy spectrum is expected to tend to the quasi-universal "Kolmogorov 5/3 law" (Kolmogorov 1941):

$$
E_{R}(k)=C_{K} \epsilon^{2 / 3} k^{-5 / 3}
$$

[later generalized by Kraichnan (1967) and Batchelor (1969) to two-dimensional turbulence, and hereafter referred to as "KBK"], where $C_{K}$ is a universal dimensionless constant $\simeq 4-6$ and $k$ is the total (isotropic) wavenumber $\left(\right.$ in $\left.^{-1}\right)$. In the direction perpendicular to the zonal flow (i.e., increasing latitude on a spherical planet, or cylindrical radius as here), however, various studies have suggested that the spectrum may equilibrate toward a different, and significantly steeper, form,

$$
E_{Z}(k)=C_{Z} \beta^{2} k^{-5},
$$

(where $C_{Z}$ is another universal dimensionless constant $\simeq 0.3-0.5$ ) provided certain conditions are fulfilled (see Galperin et al. 2006 for further discussion). Such conditions mainly relate to the respective sizes of the scales of energy injection (which need to be small enough) and those scales affected by large-scale energy removal [i.e., at wavenumbers less than the so-called frictional wavenumber $k_{\text {fr }}$; see Galperin et al. (2006) and references therein].

\section{a. Spectral slopes and anisotropies}

In the present series of experiments, the previous sections have indicated that the convectively driven turbulent flows result in the formation of jetlike, zonally elongated structures that appear to be the result of upscale nonlinear interactions, including strong and coherent interactions with the azimuthally symmetric component of the zonal flow. It is of interest, therefore, to examine whether the kinetic energy spectra reveal any similarities with those obtained in idealized numerical model studies.

Figure 12 shows kinetic energy spectra for each of the three main cases discussed above, illustrating the influence of increasing $\beta$ on the equilibrated energy spectra. In the flat bottom experiment (Fig. 12a), the total and nonzonal spectra appear to follow closely a $-5 / 3$ slope over the full range of wavenumbers from around 5-50 $\operatorname{rad~} \mathrm{m}^{-1}$, beyond which the spectrum steepens for $k>$ 50. The zonal spectrum in fact follows a slope close to $-8 / 3$, broadly consistent with an isotropic energy spectrum, with no evidence of any steepening at the lowest wavenumbers accessible in these measurements. The amplitude of the total energy spectrum is approximately $40 \%$ below the expected KBK spectrum for $C_{K}=5$ using the value of $\epsilon$ estimated from $\epsilon \simeq E_{k} / \tau_{E}$, though the reason for this is not clear.

Figure $12 \mathrm{~b}$ shows the intermediate $\beta$ case with the full sloping bottom but half the rotation rate $\Omega$. In this case the nonzonal and total energy spectra have a somewhat steeper slope than $-5 / 3$ over the range $5 \leq$ $k \leq 50 \mathrm{rad} \mathrm{m}^{-1}$, though with an amplitude approaching that of the expected KBK spectrum around $k \leq 10 \mathrm{rad}$ $\mathrm{m}^{-1}$. In contrast to case I, however, the zonal spectrum exhibits a $-8 / 3$ slope only for $k>9 \mathrm{rad} \mathrm{m}^{-1}$, becoming significantly steeper for lower values of $k$. This indi- 

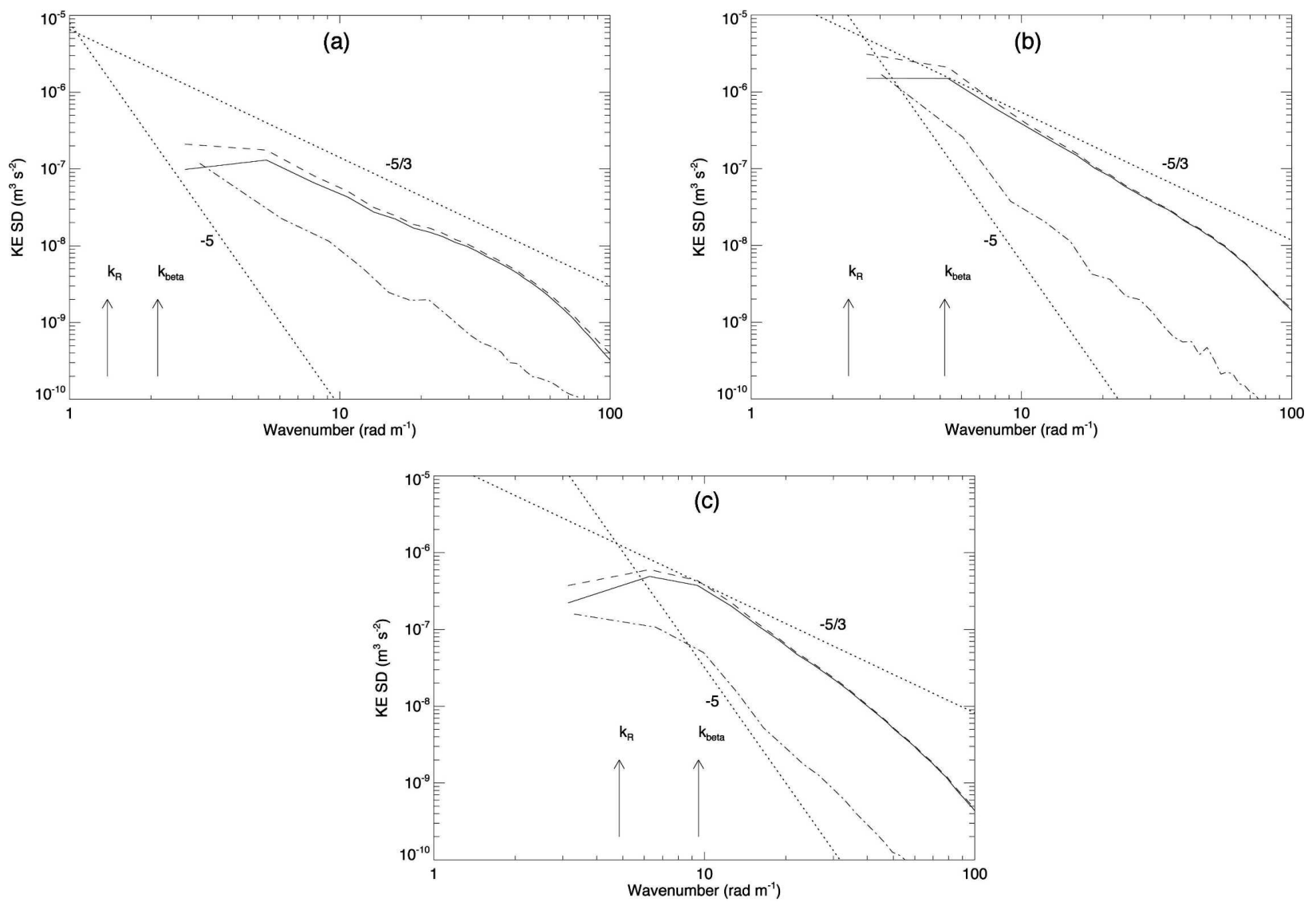

FIG. 12. Kinetic energy spectra for the three main cases (a) I, (b) II, and (c) III. Graphs show the nonzonal isotropic kinetic energy (solid lines), total kinetic energy (dashed lines), and the kinetic energy of the azimuthal mean flow (dashed-dotted lines), all in units of energy per unit mass per unit wavenumber as a function of wavenumber $\left(\mathrm{rad} \mathrm{m}^{-1}\right)$. Dotted straight line segments indicate the spectral slopes of $-5 / 3$ and -5 . The lines labeled -5 indicate segments of spectra given by Eq. (10) and those labeled $-5 / 3$ by Eq. (9) with $C_{K}=5$ in both slope and magnitude. Data were obtained by CIV analyses of images from the narrow-angle camera.

cates that the kinetic energy distribution is starting to become somewhat anisotropic at low wavenumbers, at least qualitatively consistently with numerical studies on the $\beta$ plane. It is of interest to note that the steepening of the zonal spectrum occurs around a wavenumber comparable to or somewhat greater than $k_{\beta}(\sim 5.5$ $\mathrm{rad} \mathrm{m}^{-1}$ for this flow). At the lowest wavenumbers, the nonzonal spectrum clearly flattens for $k \leq 5 \mathrm{rad} \mathrm{m}^{-1}$. Spectra obtained from wide-angle images (not shown) indicate a flattening of the nonzonal spectrum around $k$ $\sim 3 \mathrm{rad} \mathrm{m} \mathrm{m}^{-1}$, indicating a value for $k_{\mathrm{fr}}$ of around $3 \mathrm{rad}$ $\mathrm{m}^{-1}$, which is comparable to the radial wavenumber of the zonal jets.

The strongest $\beta$ case in Fig. 12c shows some even clearer signs of anisotropic structure, though evidence for the classical $\mathrm{KBK}-5 / 3$ slope is not very clear. However, the total and nonzonal spectra become tangent to the KBK form around $k=10 \mathrm{rad} \mathrm{m}^{-1}$, and in corresponding spectra obtained using the wide-angle camera (not shown) there is clearer evidence for a segment of the spectrum with a slope of $-5 / 3$ for $\sim 6 \leq k \leq 12 \mathrm{rad}$ $\mathrm{m}^{-1}$. The nonzonal and total energy spectra appear to rise to a peak at around $k=6 \mathrm{rad} \mathrm{m}^{-1}$, flattening or even decreasing as $k$ decreases below $\sim 6 \mathrm{rad} \mathrm{m}^{-1}$, again suggesting a value for $k_{\mathrm{fr}}$ of around $6 \mathrm{rad} \mathrm{m}^{-1}$, which, as in case II, corresponds closely to the radial wavenumber of the zonal jets. At smaller scales $(k>50$ rad $\left.\mathrm{m}^{-1}\right)$ the spectrum steepens, perhaps approaching the -3 slope characteristic of an enstrophy-cascading range in two-dimensional turbulence [sometimes known as Kraichnan's -3 law; Kraichnan (1967)]. At these scales, however, corresponding to a horizontal wavelength of $<10 \mathrm{~cm}$, the flow is almost certainly dominated by three-dimensional baroclinic effects, and perhaps even viscous effects at the smallest scales. The azimuthal mean spectrum is significantly steeper than $-8 / 3$ for $6<k<15 \mathrm{rad} \mathrm{m}^{-1}$, indicating fairly strongly anisotropic structure biased toward accumulation of en- 
ergy in zonal modes at low wavenumber. We plot in Fig. 12c a straight line segment with the slope -5 and magnitude consistent with Eq. (10) for comparison with the measured zonal spectrum. In this case both the slope and the magnitude of the zonal spectrum are actually comparable with the theoretical spectrum suggested, for example, from the model studies of Huang et al. (2001) and Sukoriansky et al. (2002). Such a trend is somewhat evident for case II, though not at all for case I.

The role of wind stress in adding a directly driven component of the zonal flow in each case here may partly underlie why the effects of anisotropic inertial energy cascades are difficult to identify in those cases where $\beta$ effects are relatively weak. It was mentioned above that wind stresses may be partly responsible for the presence of predominantly retrograde azimuthal flow near the outer boundary of the experiment. It is only in the case with the strongest $\beta$ effect that a possible influence of the anisotropic energy cascade becomes apparent in the zonal energy spectrum, even though some weak indication of zonation in the azimuthal flow is apparent in the intermediate $\beta$ case II.

\section{b. Spectral transfer rates}

The evolution of the anisotropic spectra discussed above is determined by the nature of the nonlinear interactions between various wavenumber components in the flow. Thus far we have concentrated on examining some of these interactions in the physical domain, by examining directly the energetic exchanges between the azimuthal mean (zonally symmetric) flow and the nonaxisymmetric eddies. However, most theoretical treatments of energy cascades consider exchanges within the spectral domain, by examining the transfer of energy from one region of the spectrum to another in wavenumber space. Anisotropy is then revealed in the channeling of energy in particular directions in wavenumber space.

Chekhlov et al. (1996), for example, computed a twodimensional enstrophy transfer function in Fourier (wavenumber) space from a sequence of direct numerical simulations of $\beta$-plane turbulence. Starting with the enstrophy evolution equation,

$$
\left(\frac{\partial}{\partial t}+2 \nu k^{2}\right) \Xi(\mathbf{k}, t)=\mathcal{T}_{\Xi}(\mathbf{k}, t),
$$

where $\Xi(\mathbf{k}, t)$ is the vorticity correlation function, $\nu$ is the kinematic viscosity of the fluid, $\mathbf{k}$ is the horizontal wave vector, and $k$ its magnitude. The spectral enstrophy transfer, $\mathcal{T}_{\Xi}(\mathbf{k}, t)$, is then given by

$$
\begin{aligned}
\mathcal{T}_{\Xi}(\mathbf{k}, t)= & 2 \pi k \mathcal{R}\left[\int_{\mathbf{p}+\mathbf{q}=\mathbf{k}} \frac{d \mathbf{p} d \mathbf{q}}{4 \pi^{2}}\right. \\
& \left.\times \frac{\mathbf{p} \times \mathbf{q}}{|\mathbf{p}|^{2}}\langle\zeta(\mathbf{p}, t) \zeta(\mathbf{q}, t) \zeta(-\mathbf{k}, t)\rangle\right],
\end{aligned}
$$

where $\zeta(\mathbf{k}, t)$ is the relative vorticity as a function of wavenumber $\mathbf{k}$ at time $t, \mathbf{p}$ and $\mathbf{q}$ are wave-vector variables of integration, $[\mathcal{R}$ ] denotes the real part, and angle brackets denote the ensemble average. To compute a spectral energy transfer map in $\mathbf{k}$ space, a cutoff wavenumber $k_{c}$ was introduced such that the energy injection into mode $\mathbf{k}$ is taken into account only via triads involving other modes with $|\mathbf{k}|>k_{c}$. The k-dependent net time-averaged enstrophy transfer into mode $\mathbf{k}$, $\mathcal{T}_{\Xi}\left(\mathbf{k} \mid k_{c}\right)$, was then computed for each mode $\mathbf{k}$ such that $|\mathbf{k}|<k_{c}$ (the so-called explicit modes), by integrating Eq. (12) over all other modes with wave vectors $\mathbf{p}$ and $\mathbf{q}$ for which $|\mathbf{p}|$ and $|\mathbf{q}|>k_{c}$ (the "implicit" modes), replacing the ensemble average with the time-mean spectral covariance. The corresponding time-averaged energy transfer function $\mathcal{T}_{E}\left(\mathbf{k} \mid k_{c}\right)$ can then be computed from

$$
\mathcal{T}_{E}\left(\mathbf{k} \mid k_{c}\right)=\frac{\mathcal{T}_{\Xi}\left(\mathbf{k} \mid k_{c}\right)}{|\mathbf{k}|^{2}} .
$$

Chekhlov et al. (1996) and Galperin et al. (2006) computed the integral in Eq. (12) for their run, designated "DNS3," and this clearly showed a strong tendency for energy to accumulate into particular wavenumbers along the $k_{y}$ axis, consistent with the formation of strong zonal jets with meridional wavenumbers $<k_{c}$.

In the present experiments, we have computed the same spectral energy transfer function in two dimensions, using the measured vorticity fields at middepth discussed above. Figure 13 shows two examples of such functions, computed from sequences of vorticity fields derived from images from the narrow-angle camera. Figure 13a shows the resulting kinetic energy transfer function for the flat bottom (case I), which indicates a general trend for upscale energy transfer toward the low wavenumber region around $|\mathbf{k}|=5-10 \mathrm{rad} \mathrm{m}^{-1}$. There is also apparently a weak tendency for the accumulation of kinetic energy into two peaks at $\mathrm{k}$ around $(\mp 3, \pm 3) \mathrm{rad} \mathrm{m}^{-1}$. This is consistent with the form of the velocity fields within this field of view shown above, with persistent diagonal flows across the domain in association with a near-stationary domain-filling eddy with a diameter $\geq 2 \mathrm{~m}$.

For the sloping boundary, on the other hand, a clearer pattern is found to emerge. Figure $13 \mathrm{~b}$ indicates a clear tendency for kinetic energy to accumulate from $|\mathbf{k}|>k_{c}$ into a roughly circular ring in wavenumber 
(a)

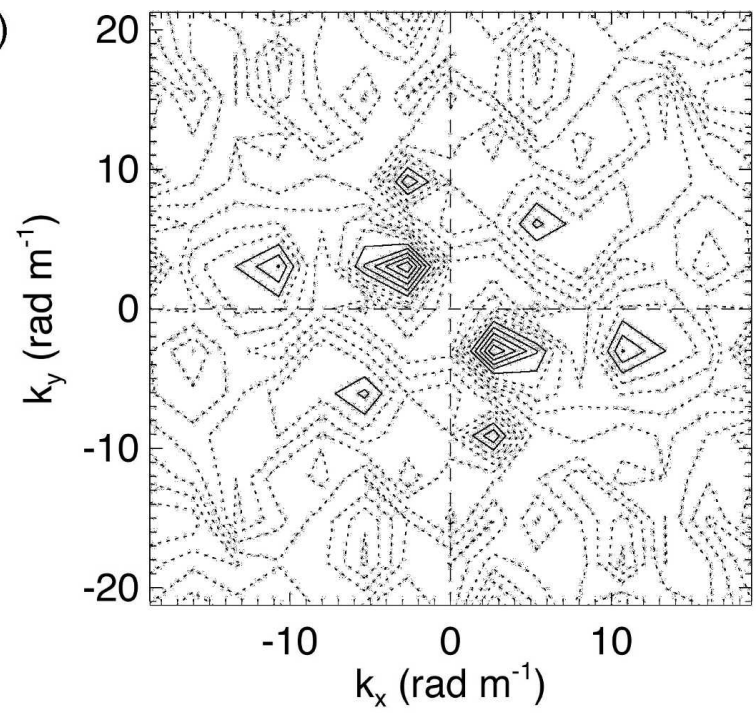

(b)

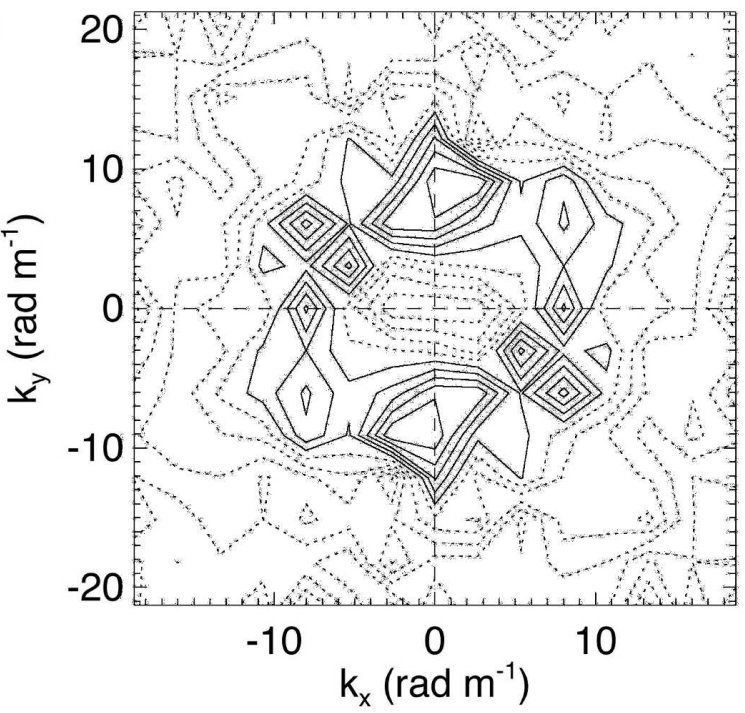

FIG. 13. Normalized spectral energy transfer functions for cases (a) I (flat bottom) and (b) III (sloping bottom, full $\beta$ ). Functions were computed from sequences of vorticity fields obtained from narrow-angle camera images, and integrated over triangles in wavenumber for $k_{c} \simeq 20 \mathrm{rad} \mathrm{m}^{-1}$, averaging in time over approximately $40-50$ rotation periods. The field of $\mathcal{T}_{E}\left(\mathbf{k} \mid k_{c}\right)$ is normalized in each case by the maximum value in the field. Contours are shown with interval 0.1 and contours below 0.5 are dashed.

space with a radius of around $8-10 \mathrm{rad} \mathrm{m}^{-1}$, but with clear maxima close to the $k_{y}$ axis at $k_{y}= \pm 8 \mathrm{rad} \mathrm{m}^{-1}$. The maxima are roughly twice the strength of the isotropic ring in $\mathcal{T}_{E}\left(\mathbf{k} \mid k_{c}\right)$, indicating a modest accumulation rate of kinetic energy into zonally elongated structures with a wavelength of a little less than $1 \mathrm{~m}$. Such a wavelength is roughly consistent with the development of the zonal jets described above, though indicates a much weaker tendency for strong jet formation than in the much clearer example presented in the numerical
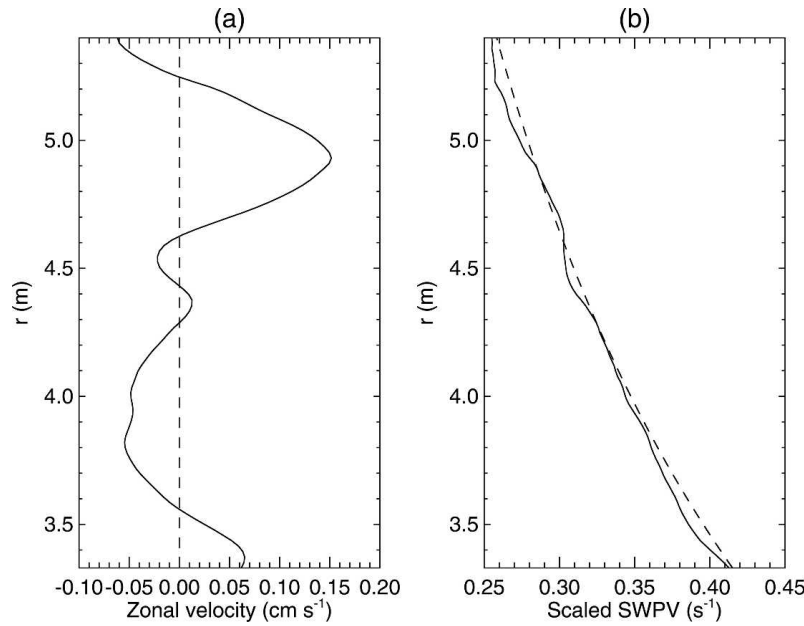

FIG. 14. (a) Radial profiles of the azimuthal and time-mean azimuthal velocity and (b) the corresponding profiles of (shallow water) potential vorticity (see text), averaged over a period of approximately $1 \mathrm{~h}$ from velocity fields acquired from the narrowangle camera.

model simulations of Chekhlov et al. (1996) and Galperin et al. (2006).

\section{Potential vorticity dynamics}

Potential vorticity (PV) almost certainly plays at least as important a role in the dynamics of the observed jets and vortices in the experiment as their energetics. In particular, the formal stability of the observed flows is generally expressed using criteria formulated in terms of potential vorticity and its gradient. In this section, therefore, we compute diagnostics relating to a potential vorticity $q$ based on a barotropic (shallow water) formulation

$$
q=\frac{2 \Omega+\zeta}{h(r)},
$$

where $\zeta$ is the relative vorticity measured at middepth. This can be determined relatively easily using the vorticity fields measured via CIV and knowledge of the variation of the depth of the tank due to the sloping lower and upper boundaries.

\section{Rayleigh-Kuo stability}

Figure 14 shows radial profiles of the time and azimuthal mean azimuthal velocity and corresponding potential vorticity, averaged over approximately $1 \mathrm{~h}$ of the late phase of the experiment. From these profiles, it is clear that there are some zonal jetlike structures that survive the time averaging, but the potential vorticity profile associated with this profile of $\bar{u}$ is evidently 
(a)

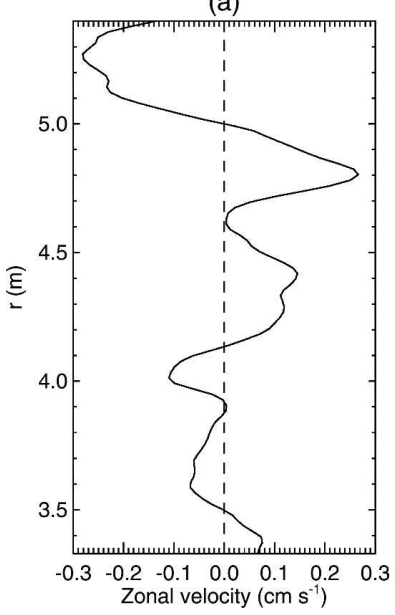

(b)

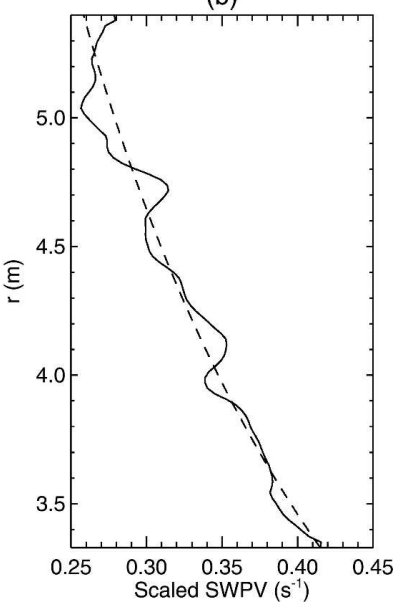

FIG. 15. (a) Radial profiles of the azimuthal and time-mean azimuthal velocity and (b) the corresponding profiles of (shallow water) potential vorticity (see text) for a typical instantaneous flow from case III in the rotating tank with sloping bottom. Velocity fields were acquired from the narrow-angle camera.

monotonic in radius. Such a result would suggest that the spatiotemporal mean azimuthal flow is consistent with a state of neutral stability with respect to the Rayleigh-Kuo criterion for barotropic stability.

On shorter time scales, however, the stability status of the azimuthal flow profile is less clear. Figures 15 and 16 show the equivalent profiles of $\bar{u}(r)$ and $\bar{q}(r)$ for an example of an instantaneous flow (Fig. 15) and a shorter-term time average (this time over just $300 \mathrm{~s}$; Fig. 16). In the first case, the instantaneous profile of $\bar{u}$ shows a stronger and more complex array of zonal jets in association with a $q$ profile that is clearly nonmonotonic. Indeed, $\partial q / \partial r$ clearly changes sign in Fig. $15 b$ several times, indicating positive violations of the Rayleigh-Kuo stability criterion consistent with active barotropic instability. Sharp positive gradients in $\bar{q}$ occur in association with prograde peaks in $\bar{u}$, while weak or reversed gradients in $\bar{q}$ are found associated with retrograde azimuthal jets. This is much as found in idealized models of active Rossby wave critical layers (see, e.g., Haynes 1989). Similar traits are also found in the PV structure of Jupiter's atmosphere (Read et al. 2006) in association with the cloud-level zonal jets.

If the profiles are averaged over an intermediate time scale, however, the violations of the Rayleigh-Kuo stability criterion are less marked but still evident, with clear indications of a PV "staircase" with radius. Figures 16a,b show profiles averaged over an interval of just $300 \mathrm{~s}$ around the time of the instantaneous flow in Figs. 15a,b. This would seem to indicate a tendency for persistent barotropic instability on time scales of a few
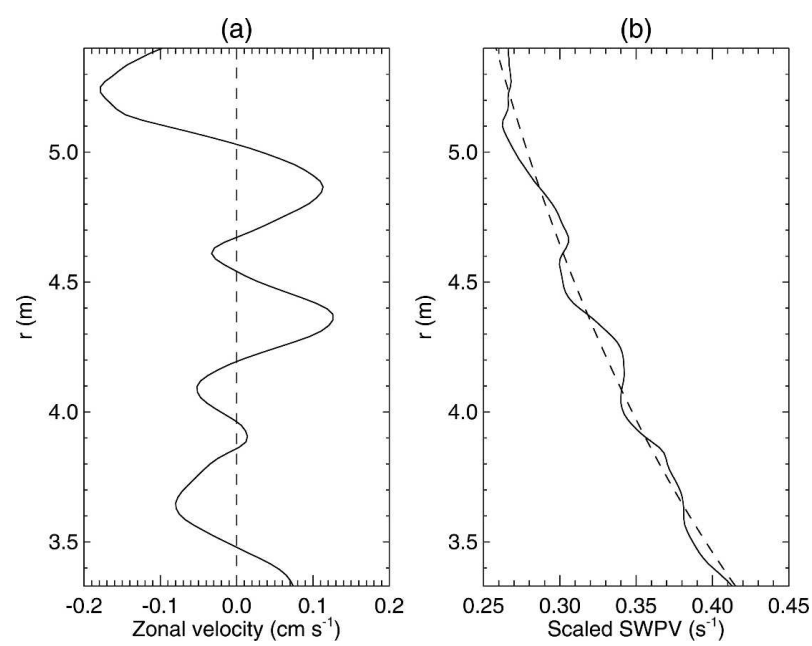

FIG. 16. (a) Radial profiles of the azimuthal and time-mean azimuthal velocity and (b) the corresponding profiles of (shallow water) potential vorticity (see text) for a flow averaged over a period of $300 \mathrm{~s}$ around the time of the snapshot in Fig. 15. Velocity fields were acquired from the narrow-angle camera.

hundred seconds, beyond which the instability is able to eliminate the regions of reversed $\partial q / \partial r$ in the PV "hyperstaircase" found on short time scales. Such a process would constitute a form of "barotropic adjustment," in which the time-averaged flow on long time scales approaches a state of near-neutral stability with regard to PV gradients. The mechanism for this is almost certainly a manifestation of a form of Rossby wave breaking, in which PV gradients become temporarily reversed as waves reach a large enough amplitude to overturn.

\section{Discussion}

Our objectives in carrying out this experiment were to seek to create conditions in the laboratory that could capture, at least qualitatively, some aspects of the dynamical regime that might characterize the banded circulation observed in the gas giant planets, and perhaps the recently discovered zonally banded currents in the earth's oceans. Such a goal turns out to be far from trivial, since (as we discuss in the following) the relevant regime is difficult to achieve on a laboratory scale because of various conflicting scaling requirements. The present experiments were made possible as a result of an all-too-brief opportunity (for six weeks in 2002) to carry out a collaborative investigation with the team at the Coriolis facility in Grenoble, which provided the best available resources to access the conditions necessary for nonlinear zonation. However, the difficulties of such an experiment should not be underestimated, and 
TABLE 2. Derived parameters for the three cases I-III. All wavenumber scales shown are in units of radians per meter. See text for explanation of symbols.

\begin{tabular}{lcccccccc}
\hline \hline Case & Ro* & $\epsilon\left(\mathrm{m}^{2} \mathrm{~s}^{-3}\right)$ & $k_{\xi}$ & $k_{\beta}$ & $k_{\mathrm{fr}}$ & $k_{R}$ & $k_{d}$ & $k_{\mathrm{dext}}$ \\
\hline I & $2.2 \times 10^{-3}$ & $1.5 \times 10^{-9}$ & 49 & 2.1 & $<3$ & 1.4 & $>13$ \\
II & $1.3 \times 10^{-2}$ & $1.1 \times 10^{-8}$ & 20 & 5.2 & $\sim 3$ & 2.3 & $>6.5$ & 0.08 \\
III & $3.4 \times 10^{-3}$ & $6.6 \times 10^{-9}$ & 61 & 9.5 & $\sim 6$ & 4.9 & $>13$ & 0.16 \\
\hline
\end{tabular}

our experimental program in the end was able to sample adequately only a few points in the parameter space of the system. Nevertheless, from the results we were able to obtain clear evidence of the processes anticipated, in association with a form of small-scale convective forcing that is as close as possible to a "natural" means of flow excitation as could be envisioned within a terrestrial laboratory. An analysis of the properties of these flows obtained in the laboratory, therefore, ought to provide important insights into the dynamical mechanisms at work within such a regime, and to allow us to put several previously proposed mechanisms for zonal jet formation and maintenance to some severe experimental tests.

\section{a. Zonostrophic regime}

Galperin and coworkers (Sukoriansky et al. 2002; Galperin et al. 2006) have attempted to define such a regime, dominated by the nonlinear generation of zonal jets (termed the "zonostrophic" regime), based on extensive studies using idealized barotropic numerical model simulations, as one in which (a) the flow is forced on relatively small scales (compared with those of the physical domain); (b) an inertial range exists over a large enough range of scales such that the $\beta$ effect can exert sufficient influence on the flow to allow anisotropic interactions to develop fully; and (c) large-scale "friction" (i.e., removal of energy at large scales) is sufficiently small to permit (b), yet large enough to prevent the accumulation of energy in the largest (domain filling) modes of the system.

The existence of such a "universal" regime has been disputed, for example, by Danilov and Gurarie (2002), though subsequently Galperin et al. (2006) and Sukoriansky et al. (2007) have claimed that the cases considered by Danilov and Gurarie (2002) did not lie in the appropriate region of parameter space. Galperin et al. (2006) proposed a series of chain inequalities as a means of formulating a set of criteria for identifying the range of parameters over which such a regime might occur. In the present context, these can be expressed in the form

$$
k_{\xi} \geq 2 k_{\beta} \geq 8 k_{\mathrm{fr}} \geq \frac{30 \pi}{L},
$$

where $k_{\xi}$ is a wavenumber $\left(\mathrm{rad} \mathrm{m}^{-1}\right)$ characteristic of the scales of energy input/forcing, $k_{\beta}$ is defined as

$$
k_{\beta}=\left(\frac{\beta^{3}}{\epsilon}\right)^{1 / 5}
$$

(where $\epsilon$ is the rate of input of kinetic energy into the upscale cascade by the forcing, $\mathrm{m}^{2} \mathrm{~s}^{-3}$ ) and represents the scale at which the Rossby wave period is comparable with the eddy overturning time scale, $k_{\mathrm{fr}}$ is the "frictional" wavenumber determined by the balance between upscale energy transfer and energy removal at large scales, and $L$ represents the physical horizontal scale of the domain (in the present case the radius of the apparatus $L=6.5 \mathrm{~m}$ ). Given sufficient time to evolve, flows that satisfy Eq. (15) are expected to develop self-similar energy spectra that adopt the universal forms given in Eq. (9) (over most angles $\phi$ ) and Eq. (10) (close to $\phi= \pm \pi / 2$ ) over discernible regions of their spectra. Under such circumstances, the zonal component of the energy spectrum [given by Eq. (10)] can be integrated over wavenumber and shown (e.g., Galperin et al. 2001,2006$)$ to lead to $k_{\mathrm{fr}} \simeq k_{R}$ to within a factor $O(1)$, where

$$
k_{R}=\left(\beta / U_{\mathrm{rms}}\right)^{1 / 2}=\frac{\pi}{L_{R}}
$$

is the Rhines wavenumber [cf. Eq. (5)]. Thus, in this regime $k_{R}$ and $\mathrm{k}_{\mathrm{fr}}$ are more or less interchangeable as estimates of the meridional scale favored by the flow for the emergence of banded zonal jets. Most recently, Sukoriansky et al. (2007) have further elucidated this relationship between $k_{\mathrm{fr}}$ and $k_{R}$, noting that forceddissipative flows for which $k_{\beta} \geq 2 k_{R}$ will exhibit most of the salient properties of the zonostrophic regime, while those for which $k_{\beta} \leq 1.5 k_{R}$ will lie in the so-called frictionally dominated regime and will not exhibit significant zonostrophic inertial ranges.

Table 2 presents estimates of the main parameters invoked in Eq. (15), derived from the experimental parameters noted in Table 1 and the apparatus dimensions, $k_{\xi}$ is estimated as $2 \pi / l_{\text {rot }}$, where $l_{\text {rot }}$ is computed using Eq. (6), and $k_{\beta}$ is obtained from Eq. (16). Note that the estimates of $\epsilon$ used here were obtained as $\epsilon \simeq$ 
$U_{\mathrm{rms}}^{2} / 2 \tau_{E}$, where $\tau_{E}$ is the Ekman spindown time scale $(=h / \sqrt{\nu \Omega})$ (see Table 1$)$. This typically results in a value for $\epsilon$ around $3 \%-15 \%$ of the input buoyancy flux $F_{B}$, which is not unreasonable since it is likely that only a small fraction of the energy input associated with $F_{B}$ will actually be utilized in the nonlinear inverse energy cascade [cf. the estimates used by Galperin et al. (2006) for the oceans and gas giant planets]. Here $k_{\text {fr }}$ represents the dominant wavenumber at which large-scale friction exerts a strong influence on the energy spectrum, and is estimated from the low wavenumber peak or break in the total or zonal wavenumber spectrum for each case.

From these estimates, it is apparent that, although each experiment satisfies the first inequality in Eq. (15), cases I and II do not rigorously satisfy either of the remaining two criteria for the zonostrophic regime. Case III, however, with the strongest $\beta$ effect, comes the closest to fulfilling all of the criteria, with $k_{\beta} \simeq 2 k_{R}$. For case II, our estimates of $k_{\beta}$ and $k_{R}$ are probably too close together to allow much of an inertial range to develop between them, placing it on the margins of the zonostropic and frictionally dominated regimes. As discussed further by Galperin et al. (2006), this section of the spectrum is critical for the emergence of the fully formed $k^{-5}$ spectrum in the zonal flow, and the separation of $k_{\beta}$ and $k_{\mathrm{fr}}$ or $k_{R}$ is much greater for the outer gas giant planets than could be obtained in our experiments here. This may well help to account for why our banded zonal jets are much less dominant in the laboratory than are typical in the atmospheres of Jupiter and Saturn. Even so, the flows obtained in case III do show a number of features suggestive of the zonostrophic regime, indicating that we were able to enter at least the margins of this class of dynamical behavior. Galperin et al. (2006) note that the oceans also do not quite satisfy the criteria on $k_{\beta} / k_{\mathrm{fr}}$ set out in Eq. (15), though they fulfill them somewhat more clearly than in our experiment III.

Also included in Table 2 are estimates of wavenumber scales based on both the internal and external deformation radii. In Eq. (4) $k_{d}$ is defined, though only a lower limit can be estimated because $N$ is poorly defined. The external deformation scale is estimated from $k_{\text {dext }} \sim f / \sqrt{g \bar{h}}$, though this wavenumber turns out to be much less than the smallest wavenumber that can be accommodated in the annular domain. These estimates suggest that the external deformation radius does not play a role in the dynamics of this experiment, but internal baroclinic disturbances could be significant. However, there is no evidence of scales of motion that manifest themselves as different from those represented by $k_{\beta}, k_{R}$, or $k_{\xi}$.

\section{b. Scales and spectral transfers}

Insofar as our experiment III does lie parametrically in the zonostrophic regime, therefore, the diagnostics computed above help to reveal the dynamical mechanisms at work in sustaining a zonally banded circulation. We have shown that, provided $\beta$ is strong enough, a flow excited by stirring at small scales will evolve a large-scale barotropic zonation, accompanied by an ensemble of barotropic vortical structures, many of which propagate zonally, at least qualitatively, like Rossby waves. The formation of the zonation in azimuthal flow appears to be largely as a result of direct eddy-zonal flow interactions, with a clear tendency for kinetic energy to accumulate upscale into the form of zonally elongated structures or jets. Such a tendency is at least consistent with the hypothesis of Balk (2005) that such zonally elongated structures will be favored in flows constrained simultaneously to conserve the three dynamical invariants: energy, enstrophy and the geometric invariant noted by Balk (1991) for Rossby wave systems. It would be of interest in future work to investigate further the latter invariant in actual diagnostics of actual flows and numerical simulations, and to examine in greater depth the validity of this hypothesis.

The scale favored by the zonation process is a significant factor in determining the effect of zonostrophic anisotropy on flow structure. The Rhines wavenumber, $k_{R}$ [as defined in Eq. (17)] has often been suggested as representing the likely scale of zonal jet formation. As originally noted by Hide (1966), such a scale represents a rough balance between relative and planetary vorticity advection, though this may not be the most useful balance to consider. Galperin et al. (2001) put forward an alternative interpretation, relating the favored jet scale, represented by $k_{\text {fr }}$, to a balance between upscale energy transfer and large-scale energy removal. Both of these are effectively internal parameters, dependent on flow-dependent quantities, so are not particularly useful as predictive quantities. But they appear to be broadly consistent with the observed scale of jets found in the present experiments. However, our experiments II and III indicate in this case that $k_{\mathrm{fr}} \simeq 1.5 k_{R}$, which is not quite as suggested by Galperin et al. (2001) and Galperin et al. (2006) for the zonostrophic regime, though is close to the form suggested by Sukoriansky et al. (2007). In both cases II and III the scale $k_{\text {fr }}$ corresponds quite closely to the radial wavenumber of the observed zonal jets, indicating that the scale separating adjacent zonal jets is reasonably well estimated by $\sim 1.5 k_{R}$. The Rhines scale $k_{R}$ also seems to be quite closely consistent with observed flows in the outer planets (e.g., Vasavada and Showman 2005) and the oceans 
(Richards et al. 2006), which satisfy Eq. (15) even more closely.

An important aspect of the near-equivalence of $k_{\mathrm{fr}}$ with $k_{R}$, asserted by Galperin et al. (2001), Galperin et al. (2006), and Sukoriansky et al. (2007), is the assumption that energy removal takes place in a relatively scale-independent manner. This is consistent, for example, with Ekman damping, such as would apply to our present experiments, and in the earth's oceans. The principal mechanism for energy removal on large scales on the gas giant planets, however, is not clear at the present time. None of the gas giants is believed to possess a solid underlying surface, so the relevance of Ekman damping seems unlikely. But presumably there must be some means of removing barotropic kinetic energy from the flow on large scales that, given the apparent consistency of the observed jets with the Rhines wavenumber, must act comparably on all scales. This is an aspect of the energy flow within the atmospheric circulation of these planets that deserves closer attention in future work.

\section{c. Convective forcing}

The method of forcing used herein seems to work well as a "natural" means of injecting energy at small scales, though the use of salt-driven convection does pose significant practical difficulties in sustaining a uniform and steady input of energy over long periods of time. In future experiments, it would be useful to explore the possibility of driving convection via imposed thermal contrasts, although providing sufficient heating and cooling (on the order of $10-100 \mathrm{~W} \mathrm{~m}^{-2}$ on the scale of the Coriolis facility) would be a major undertaking. The imposition of unstable stratification has clear parallels with what may be happening in the atmospheres of the gas giant planets; though in their case, the role of moist convective processes needs to be taken into account (Gierasch et al. 2000; Ingersoll et al. 2000). The latter probably concentrates intense convection into small and highly intermittent regions though would certainly operate on a small scale compared with that of the planet itself. But an overall upwelling energy input of around 5-6 $\mathrm{W} \mathrm{m}^{-2}$ from the interior heat source on Jupiter (e.g., Vasavada and Showman 2005), coupled with a depth for convection of at least $50 \mathrm{~km}$, would suggest an average natural Rossby number Ro* of 0.1 or less, placing it in a qualitatively similar convective regime to the present experiments.

Similar types of deep, rotationally controlled convective forcing may also occur in the oceans (Marshall and Schott 1999), though only in very restricted regions such as the polar oceans and the Mediterranean. More commonly, it is baroclinic instability on horizontal scales of 50-100 $\mathrm{km}$ that provides the dominant source of "stirring" in the wider ocean basins, and may be responsible for the energy input for the putative smallscale zonal jets now being found in ocean models and observations (Galperin et al. 2004; Maximenko et al. 2005; Richards et al. 2006). This was the original concept for exciting geostrophic turbulence by Salmon (1980), and there is certainly some evidence for zonation in small-scale laboratory experiments on baroclinic instability using sloping endwalls (Mason 1975; Bastin and Read 1998), which would support the notion that the zonostrophic regime does not depend strongly on the mechanism of small-scale energy injection. But laboratory experiments to date on baroclinic instability with sloping endwalls have been carried out only on a relatively small scale, and almost certainly have not satisfied the criteria expressed in Eq. (15). It would be of significant interest to investigate this further on the scale of the Coriolis facility.

\section{d. Potential vorticity}

The potential vorticity structure of the equilibrated banded zonal flow shows some clear signatures of intense interactions with eddies. Although the net effect of such interactions is to accelerate the zonal flow and to modify its structure, especially in the vicinity of Rossby critical layers that develop spontaneously as the flow evolves, the resulting instantaneous zonal flow ends up in a state that violates many of the "classical" criteria for stability. Instantaneous velocity fields show evidence for smoothing out and even reversals of the radial PV gradient, indicative of local Rossby wave breaking. Longer time averages, however, are much closer to a state of neutral stability. This would seem to suggest that barotropic instability acts as an adjustment mechanism on long enough time scales in these experiments, such that instantaneous flows are indeed unstable but adjust the PV distribution over time scales of a few hundred $\tau_{r}$ toward marginal stability. This would appear to be in contrast with results from unforced numerical model simulations, which seem to produce jets that are much closer to neutral stability, even for instantaneous fields. The continuous forcing must therefore play a major role in perturbing the zonal flow away from this marginal state, by an amount that presumably depends on the strength of forcing.

It has long been noted that the westward zonal jets on the outer planets tend to violate the classical Rayleigh-Kuo/Charney-Stern instability criteria (Vasavada and Showman 2005; Read et al. 2006), though they may be closer to neutrality with respect to Arnold's second stability theorem (Dowling 1993, 1995). This may again be a signature of the effect of the processes forcing the 
jets on Jupiter and Saturn, whose origins are still poorly understood in detail, though may lead to Rossby wave breaking as energy cascades upscale from the smallerscale convective forcing. Further studies of the relationship between eddy forcing and stability criteria in experiments and models may be a useful source of further information on these mechanisms for driving zonal flows on the outer planets. Finally, we remark that the extent to which the small-scale jets found in ocean observations and models violate the classical instability criteria has not yet been investigated in detail, but could form an important means of discriminating between zonostrophic mechanisms for jet formation and alternative mechanisms (e.g., see Berloff 2005).

Acknowledgments. We are grateful to the HYDRALAB program, funded by the EC Contract Access to Major Research Infrastructures, for their support during the experimental design and acquisition phase of this project. Additional support was provided by the U.K. Particle Physics and Astronomy Research Council. Thanks are also due to the technical team at LEGI/ Coriolis for their excellent assistance with the design and execution of the experiments, and to Profs B. Galperin and S. B. Sukoriansky for comments and discussions during the course of this study.

\section{REFERENCES}

Andrews, D. G., J. R. Holton, and C. B. Leovy, 1987: Middle Atmosphere Dynamics. Academic Press, 489 pp.

Balk, A., 1991: A new invariant for Rossby wave systems. Phys. Lett. A, 155, 20-24.

— 2005: Angular distribution of Rossby wave energy. Phys. Lett. A, 345, 154-160.

Bastin, M. E., and P. L. Read, 1998: Experiments on the structure of baroclinic waves and zonal jets in an internally heated rotating cylinder of fluid. Phys. Fluids, 10, 374-389.

Batchelor, G. K., 1969: Computation of the energy spectrum in homogeneous two-dimensional turbulence. Phys. Fluids, 12 (Suppl.), 233-239.

Berloff, P. S., 2005: On rectification of randomly forced flows. $J$. Mar. Res., 63, 497-527.

Chekhlov, A., S. A. Orszag, S. Sukoriansky, B. Galperin, and I. Staroselsky, 1996: The effect of small-scale forcing on largescale structures in two-dimensional flows. Physica D, 98, 321334.

Condie, S. A., and P. B. Rhines, 1994: A convective model for the zonal jets in the atmospheres of Jupiter and Saturn. Nature, 367, 711.

Danilov, S., and D. Gurarie, 2002: Rhines scale and the spectra of $\beta$-plane turbulence with bottom drag. Phys. Rev., E65, doi:10.1103/PhysRevE.65.0673301.

Dowling, T. E., 1993: A relationship between potential vorticity and zonal wind on Jupiter. J. Atmos. Sci., 50, 14-22.

_ 1995: Dynamics of Jovian atmospheres. Annu. Rev. Fluid Mech., 27, 293-334.

Fernando, J. S. F., R. Chen, and D. L. Boyer, 1991: Effects of rotation on convective turbulence. J. Fluid Mech., 228, 513547

Fincham, A. M., and G. R. Spedding, 1997: Low cost, high resolution DPIV for measurement of turbulent fluid flow. Exp. Fluids, 23, 449-462.

_ , and G. Delerce, 2000: Advanced optimization of correlation imaging velocimetry algorithms. Exp. Fluids, 29 (Suppl.), S13-S22.

Galperin, B., S. Sukoriansky, and H.-P. Huang, 2001: Universal $\mathrm{n}^{-5}$ spectrum of zonal flows on giant planets. Phys. Fluids, 13, $1545-1548$

- H. Nakano, H.-P. Huang, and S. Sukoriansky, 2004: The ubiquitous zonal jets in the atmospheres of giant planets and Earth's oceans. Geophys. Res. Lett., 31, 13303, doi:10.1029/ 2004GL019691.

- -, S. Sukoriansky, N. Dikovskaya, P. L. Read, Y. H. Yamazaki, and R. Wordsworth, 2006: Anisotropic turbulence and zonal jets in rotating flows with a $\beta$ effect. Nonlinear Processes Geophys., 13, 83-98.

García-Melendo, E., and A. Sánchez-Lavega, 2001: A study of the stability of Jovian zonal winds from HST images: 1995-2000. Icarus, 152, 316-330.

Gierasch, P. J., and Coauthors, 2000: Observation of moist convection in Jupiter's atmosphere. Nature, 403, 628-630.

Haynes, P. H., 1989: The effect of barotropic instability on the nonlinear evolution of a Rossby-wave critical layer. J. Fluid Mech., 207, 231-266.

Hide, R., 1966: On the circulation of the atmospheres of Jupiter and Saturn. Planet Space Sci., 14, 669-675.

Huang, H.-P., B. Galperin, and S. Sukoriansky, 2001: Anisotropic spectra in two-dimensional turbulence on the surface of a rotating sphere. Phys. Fluids, 13, 225-240.

Ingersoll, A. P., R. F. Beebe, J. L. Mitchell, G. W. Garneau, G. M. Yagi, and J.-P. Müller, 1981: Interaction of eddies and mean zonal flow on Jupiter as inferred from Voyager 1 and 2 images. J. Geophys. Res., 86, 8733-8743.

—, P. G. Gierasch, D. Banfield, and A. R. Vasavada, 2000: Moist convection as an energy source for the large-scale motions in Jupiter's atmosphere. Nature, 403, 630-632.

— , and Coauthors, 2004: Dynamics of Jupiter's atmosphere. Jupiter: The Planet, Satellites and Magnetosphere, F. Bagenal, T. Dowling, and W. McKinnon, Eds., Cambridge University Press, 105-128.

Kolmogorov, A. N., 1941: The local structure of turbulence in incompressible viscous fluid for very large Reynolds number. Dokl. Akad. Nauk SSSR, 30, 9-13.

Kraichnan, R. H., 1967: Inertial ranges in two-dimensional turbulence. Phys. Fluids, 10, 1417-1423.

Limaye, S. S., 1986: Jupiter: New estimates of the mean zonal flow at the cloud level. Icarus, 65, 335-352.

Marshall, J., and F. Schott, 1999: Open-ocean convection: Observations, theory and models. Rev. Geophys., 37, 1-64.

Mason, P. J., 1975: Baroclinic waves in a container with sloping endwalls. Philos. Trans. Roy. Soc. London, A278, 397.

Maximenko, N., B. Bang, and H. Sasaki, 2005: Observational evidence of alternating zonal jets in the world ocean. Geophys. Res. Lett., 32, L12607, doi:10.1029/2005GL022728.

Maxworthy, T., and S. Narimousa, 1994: Unsteady, turbulent convection into a homogeneous, rotating fluid, with oceanographic applications. J. Phys. Oceanogr., 24, 865-887.

Pfeffer, R. L., G. Buzyna, and W. W. Fowlis, 1974: Synoptic features and energetics of wave-amplitude vacillation in a rotating, differentially-heated fluid. J. Atmos. Sci., 31, 622-645. 
Porco, C. C., and Coauthors, 2003: Cassini imaging of Jupiter's atmosphere, satellites and rings. Science, 299, 1541-1547.

- - and Coauthors, 2005: Cassini imaging science: Initial results on Saturn's atmosphere. Science, 307, 1243-1247.

Read, P. L., 1985: Finite-amplitude, neutral baroclinic eddies and mean flows in an internally heated rotating fluid: 1 . Numerical simulations and quasi-geostrophic "free modes." Dyn. Atmos. Oceans, 9, 135-207.

__ 1986: Stable, baroclinic eddies on Jupiter and Saturn: A laboratory analog and some observational tests. Icarus, 65, 304-334.

— 2001: Transition to geostrophic turbulence in the laboratory, and as a paradigm in atmospheres and oceans. Surv. Geophys., 22, 265-317.

_ , Y. H. Yamazaki, S. R. Lewis, K. Miki-Yamazaki, J. Sommeria, H. Didelle, and A. Fincham, 2004: Jupiter's and Saturn's convectively-driven banded jets in the laboratory. Geophys. Res. Lett., 31, L22701, doi:10.1029/2004GL020106.

— , P. J. Gierasch, B. J. Conrath, A. Simon-Miller, T. Fouchet, and Y. H. Yamazaki, 2006: Mapping potential vorticity dynamics on Jupiter: 1. Zonal mean circulation from Cassini and Voyager 1 data. Quart. J. Roy. Meteor. Soc., 132, 15771603.

Rhines, P. B., 1975: Waves and turbulence on a $\beta$-plane. J. Fluid Mech., 69, 417-443.

Richards, K. J., N. A. Maximenko, F. O. Bryan, and H. Sasaki, 2006: Zonal jets in the Pacific Ocean. Geophys. Res. Lett., 33, L03605, doi:10.1029/2005GL024645.

Salmon, R., 1980: Baroclinic instability and geostrophic turbulence. Geophys. Astrophys. Fluid Dyn., 15, 167-211.

Salyk, C., A. P. Ingersoll, J. Lorre, A. Vasavada, and A. D. Del Genio, 2006: Interaction between eddies and mean flow in
Jupiter's atmosphere: Analysis of Cassini imaging data. Icarus, 186, 430-442.

Sánchez-Lavega, A., J. F. Rojas, and P. V. Sada, 2000: Saturn's zonal winds at cloud level. Icarus, 147, 405-420.

_ R. Hueso, S. Perez-Hoyos, and J. F. Rojas, 2006: A strong vortex in Saturn's south pole. Icarus, 184, 524-531.

Simon, A. A., 1999: The structure and temporal stability of Jupiter's zonal winds: A study of the north tropical region. Icarus, 141, 29-39.

Sromovsky, L. A., H. E. Revercomb, V. E. Suomi, S. S. Limaye, and R. J. Krauss, 1982: Jovian winds from Voyager 2. Part II: Analysis of eddy transports. J. Atmos. Sci., 39, 1433-1445.

Sukoriansky, S., B. Galperin, and N. Dikovskaya, 2002: Universal spectrum of two-dimensional turbulence on a rotating sphere and some basic features of atmospheric circulation on giant planets. Phys. Rev. Lett., 89, doi:10.1103/PhysRevLett.89. 124501.

_ , N. Dikovskaya, and B. Galperin, 2007: On the arrest of inverse energy cascade and the Rhines scale. J. Atmos. Sci., 64, 3312-3327.

Vallis, G. K., and M. E. Maltrud, 1993: Generation of mean flows and jets on a beta plane and over topography. J. Phys. Oceanogr., 23, 1346-1362.

Vasavada, A., and A. Showman, 2005: Jovian atmospheric dynamics: An update after Galileo and Cassini. Rep. Prog. Phys., 68, 1935-1996.

Williams, G. P., 1978: Planetary circulations: I. Barotropic representation of Jovian and terrestrial turbulence. J. Atmos. Sci., 35, 1399-1426.

_- 1979: Planetary circulations: II. The Jovian quasigeostrophic regime. J. Atmos. Sci., 36, 932-968.

, 2003: Jovian dynamics. Part III: Multiple, migrating and equatorial jets. J. Atmos. Sci., 60, 1270-1296. 


\section{CORRIGENDUM}

The name of Dr. Adam Fincham was inadvertently left off of the list of coauthors on the title page of "Dynamics of Convectively Driven Banded Jets in the Laboratory," which was published in the November 2007 issue of the Journal of Atmospheric Sciences and is part of the Jets and Annular Structures in Geophysical Fluids special collection. The correct full list of authors for this paper appears below.

\section{Peter L. Read, Yasuhiro H. Yamazaki, Stephen R. Lewis, ${ }^{*}$ Paul D. Williams, ${ }^{+}$ ROBIN WORDSWORTH, AND KUnIKO MiKi-YAMAZAKI \\ Clarendon Laboratory, Department of Physics, University of Oxford, Oxford, United Kingdom}

$$
\text { JoËl Sommeria, Henri Didelle, And Adam M. Fincham }{ }^{\#}
$$

LEGI Coriolis, Grenoble, France

\footnotetext{
* Current affiliation: Department of Physics and Astronomy, The Open University, Milton, Keynes, United Kingdom.

+ Current affiliation: Department of Meteorology, University of Reading, Reading, United Kingdom.

\# Current affiliation: Department of Aerospace and Mechanical Engineering, University of Southern California, Los Angeles, California.
} 